\title{
Hidden-charm tetraquarks with strangeness in the chiral quark model
}

\author{
Gang Yang $\odot,{ }^{1, *}$ Jialun Ping, ${ }^{2, \dagger}$ and Jorge Segovia $\oplus^{3, *}$ \\ ${ }^{1}$ Department of Physics, Zhejiang Normal University, Jinhua 321004, China \\ ${ }^{2}$ Department of Physics and Jiangsu Key Laboratory for Numerical Simulation of Large Scale Complex Systems, \\ Nanjing Normal University, Nanjing 210023, People's Republic of China \\ ${ }^{3}$ Departamento de Sistemas Físicos, Químicos y Naturales, \\ Universidad Pablo de Olavide, E-41013 Sevilla, Spain
}

(Received 12 September 2021; accepted 25 October 2021; published 23 November 2021)

\begin{abstract}
The hidden-charm tetraquarks with strangeness, $c \bar{c} s \bar{q}(q=u, d)$, in $J^{P}=0^{+}, 1^{+}$, and $2^{+}$are systematically investigated in the framework of real- and complex-scaling range of a chiral quark model, whose parameters have been fixed in advance describing hadron, hadron-hadron, and multiquark phenomenology. Each tetraquark configuration, compatible with the quantum numbers studied, is taken into account; this includes meson-meson, diquark-antidiquark, and K-type arrangements of quarks with all possible color wave functions in the four-body sector. Among the different numerical techniques to solve the Schrödinger-like four-body bound state equation, we use a variational method in which the trial wave function is expanded in complex-range Gaussian basis functions, which is characterized by its simplicity and flexibility. This theoretical framework has already been used to study different kinds of multiquark systems, such as the hidden-charm pentaquarks $P_{c}^{+}$and doubly charmed tetraquarks $T_{c c}^{+}$. The recently reported $Z_{c s}$ states by the BESIII and LHCb Collaborations could be associated, in our investigation, with either compact tetraquark or hadronic molecular resonance configurations. However, it is fair to recognize that our complex poles survive when either the $(c \bar{c})(s \bar{q})$ or $(c \bar{q})(s \bar{c})$ configuration is preserved, but most of them get diluted when both are considered at the same time, indicating that such states seem to be very unstable. Finally, we mention that similar types of structures are also found in the mass range between 3.8 and $4.6 \mathrm{GeV}$.
\end{abstract}

DOI: $10.1103 /$ PhysRevD.104.094035

\section{INTRODUCTION}

A structure with a significance of $5.3 \sigma$ was reported in the process of $e^{+} e^{-} \rightarrow K^{+}\left(D_{s}^{-} D^{* 0}+D_{s}^{*-} D^{0}\right)$ by the BESIII Collaboration [1]; its experimentally measured mass and width were $3982.5_{-2.6}^{+1.8} \pm 2.1$ and $12.8_{-4.4}^{+5.3} \pm 3.0 \mathrm{MeV}$, respectively. Undoubtedly, the named $Z_{c s}(3985)$ state was the first candidate of a charged hidden-charm tetraquark state with strangeness.

Later on, more charmoniumlike states with strange content were reported by the $\mathrm{LHCb}$ Collaboration in protonproton collisions [2]. The $Z_{c s}(4000)$ is observed in the $B^{+} \rightarrow J / \psi \phi K^{+}$decay, with mass and width $4003 \pm 6_{-14}^{+4}$ and $131 \pm 15 \pm 26 \mathrm{MeV}$, respectively, and the preferred spin-parity is $J^{P}=1^{+}$. The $X(4685)$, also with $J^{P}=1^{+}$ quantum numbers, decays to a $J / \psi \phi$ final state with a high

\footnotetext{
*yanggang@zjnu.edu.cn

jlping@njnu.edu.cn

;jegovia@upo.es
}

Published by the American Physical Society under the terms of the Creative Commons Attribution 4.0 International license. Further distribution of this work must maintain attribution to the author(s) and the published article's title, journal citation, and DOI. Funded by SCOAP. significance claimed by the collaboration. Furthermore, the $Z_{c s}(4220)$ and $X(4630)$ are also reported with significance exceeding $5 \sigma$ derivations. These facts trigger many theoretical investigations on the nature of hidden-charm tetraquark with strangeness.

In many theoretical works, the $Z_{c s}(3985)$ is identified as the strange partner of the $Z_{c}(3900)$ within the $S U(3)_{f}$ symmetry, and, thus, the hadronic molecular configuration is proposed. In particular, the $D^{*} \bar{D}_{s}-D \bar{D}_{s}^{*}$ and $D^{*} \bar{D}_{s}^{*}$ molecules with spin-parity $J^{P}=1^{+}$state can be related to the mentioned $Z_{c s}^{(*)}$ particles [3]. This result is also supported by a coupled-channel calculation [4], a variety of effective field theory frameworks [5-8], approaches based on QCD sum rules $[9,10]$, and potential model descriptions [11]. The authors of Ref. [12] claimed the presence of an important bare component in the resonances $Z_{c s}(3985), Z_{c}(3900)$, and $X(4020)$ when a unified study of these states was performed within the framework of an effective range expansion. Meanwhile, the two- and four-body configuration mixing scheme for describing the $Z_{c s}(3985)$ state has been proposed to be crucial in many theoretical investigations; viz. the $Z_{c s}(3985)$ is excluded as a pure $D^{* 0} D_{s}^{-} / D^{0} D_{s}^{*-} / D^{* 0} D_{s}^{*-}$ hadronic molecular state in, for instance, the one-bosonexchange model $[13,14]$ and constituent quark model [15]. 
The $Z_{c s}(3985)$ and $Z_{c s}(4003)$ can be explained well within a mixture formalism in Refs. [16,17].

Notwithstanding this, many theoretical approaches conclude that a compact tetraquark structure is also possible for the $Z_{c s}(3985)$, e.g., one-boson-exchange model [14], constituent quark model [15], relativistic quark model [18,19], and QCD sum rules [20]. Furthermore, some novel pictures for the $Z_{c s}(3985)$ state are proposed. Particularly, the $Z_{c s}(3985)$ can be identified as a reflection structure of charmed-strange meson $D_{s 2}^{*}$ (2573) [21]. It is also explained as a genuine state, either virtual or bound, in a contact potential model [22]. Additionally, its photoproduction [23], magnetic dipole moment [24], and properties in a hot dense medium $[25,26]$ have been recently discussed theoretically considering different natures for the $Z_{c s}(3985)$.

Concerning the $Z_{c s}(4000), Z_{c s}(4220), X(4630)$, and $X(4685)$ states, there are in the literature interpretations compatible with hadronic molecules [27-30], compact tetraquark structures [30-33], and even nonresonance configurations [34]. Besides, the magnetic moments of the $Z_{c s}(4000)$ and $Z_{c s}(4220)$ are calculated by means of lightcone QCD sum rules [35].

In order to disentangle the nature of these charmoniumlike resonances with strangeness announced recently by the LHCb and BESIII Collaborations, a systematical investigation on the hidden-charm tetraquarks with strange content- $c \bar{c} s \bar{q}(q=u, d)$-is performed within a chiral quark model formalism. The same theoretical framework has already been applied with success in the description of other multiquark systems, e.g., hidden-charm and -bottom pentaquarks [36,37], doubly charm pentaquarks [38], doubly heavy tetraquarks $Q Q \bar{q} \bar{q}[39,40]$, and strangeheavy tetraquarks $s Q \bar{q} \bar{q} \quad(q=u, d, s ; Q=c, b)$ [41]. Particularly, we have explained the hidden-charm pentaquarks [36], $P_{c}^{+}(4312), \quad P_{c}^{+}(4380), \quad P_{c}^{+}(4440), \quad$ and $P_{c}^{+}(4457)$, reported by the LHCb Collaboration $[42,43]$ and predicted the doubly charmed tetraquark [39] $T_{c c}^{+}$, announced very recently by the same experimental collaboration $[44,45]$. It is also worth highlighting that the same theoretical approach was previously applied to the charmonium, bottomonium, and heavy baryon sectors, studying their spectra [46-49], their electromagnetic, weak, and strong decays and reactions [50-53], and their coupling with meson-meson thresholds [54-57].

Our formulation in real- and complex-scaling method of the theoretical formalism has been discussed in detail in Ref. [58]. The complex-scaling method (CSM) allows us to distinguish three kinds of scattering singularities-bound, resonance, and scattering-which allows us to perform a complete analysis of the scattering problem within the same formalism. Furthermore, the meson-meson, diquark-antidiquark, and K-type configurations, plus their couplings, are considered for the tetraquark system. Finally, the RayleighRitz variational method is employed in dealing with the spatial wave functions of the $c \bar{c} s \bar{q}$ tetraquark states, which are expanded by means of the well-known Gaussian expansion method (GEM) of Ref. [59].

The manuscript is arranged as follows. In Sec. II, the theoretical framework is presented; we briefly describe the complex-range method applied to a chiral quark model and the $c \bar{c} s \bar{q}(q=u, d)$ tetraquark wave functions. Section III is devoted to the analysis and discussion of the obtained low-lying $c \bar{c} s \bar{q}(q=u, d)$ tetraquark states with $J^{P}=0^{+}$, $1^{+}$, and $2^{+}$and isospin $I=1 / 2$. Finally, we summarize and give some prospects in Sec. IV.

\section{THEORETICAL FRAMEWORK}

A thorough review of the theoretical formalism used herein has been recently published in Ref. [58]. We shall, however, focus on the most relevant features of the chiral quark model and the numerical method concerning the strange hidden-charm tetraquarks, viz. the $c \bar{c} s \bar{q}$ system.

Within the so-called complex-range investigations, the relative coordinate of a two-body interaction is rotated in the complex plane by an angle $\theta$, i.e., $\vec{r}_{i j} \rightarrow \vec{r}_{i j} e^{i \theta}$. Therefore, the general form of the four-body Hamiltonian reads

$H(\theta)=\sum_{i=1}^{4}\left(m_{i}+\frac{\vec{p}_{i}^{2}}{2 m_{i}}\right)-T_{\text {c.m. }}+\sum_{j>i=1}^{4} V\left(\vec{r}_{i j} e^{i \theta}\right)$,

where $m_{i}$ is the quark mass, $\vec{p}_{i}$ is the quark's momentum, and $T_{\text {c.m. }}$ is the center-of-mass kinetic energy. According to the so-called $\mathrm{ABC}$ theorem $[60,61]$, the complex-scaled Schrödinger equation

$$
[H(\theta)-E(\theta)] \Psi_{J M}(\theta)=0
$$

has (complex) eigenvalues which can be classified into three different kinds: bound, resonance, and continuum (scattering) states. Those which are either bound or resonance are independent of the rotated angle $\theta$; however, the first ones are always fixed on the coordinate axis (there is no imaginary part of the eigenvalue), whereas the second ones are located above the corresponding threshold lines with a total decay width $\Gamma=-2 \operatorname{Im}(E)$.

The dynamics of the $c \bar{c} s \bar{q}$ tetraquark system is driven by a two-body potential

$$
V\left(\vec{r}_{i j}\right)=V_{\chi}\left(\vec{r}_{i j}\right)+V_{\mathrm{CON}}\left(\vec{r}_{i j}\right)+V_{\mathrm{OGE}}\left(\vec{r}_{i j}\right),
$$

which takes into account the most relevant features of QCD at its low-energy regime: dynamical chiral symmetry breaking, confinement, and the perturbative one-gluon exchange (OGE) interaction. Herein, the low-lying $S$-wave positive parity $c \bar{c} s \bar{q}$ tetraquark states shall be investigated, and, thus, the central and spin-spin terms of the potential are the only ones needed.

One consequence of the dynamical breaking of chiral symmetry is that Goldstone boson exchange interactions 
appear between constituent light quarks $u, d$, and $s$. Therefore, the chiral interaction can be written as [62]

$$
V_{\chi}\left(\vec{r}_{i j}\right)=V_{\pi}\left(\vec{r}_{i j}\right)+V_{\sigma}\left(\vec{r}_{i j}\right)+V_{K}\left(\vec{r}_{i j}\right)+V_{\eta}\left(\vec{r}_{i j}\right)
$$

given by

$$
\begin{gathered}
V_{\pi}\left(\vec{r}_{i j}\right)=\frac{g_{c h}^{2}}{4 \pi} \frac{m_{\pi}^{2}}{12 m_{i} m_{j}} \frac{\Lambda_{\pi}^{2}}{\Lambda_{\pi}^{2}-m_{\pi}^{2}} m_{\pi}\left[Y\left(m_{\pi} r_{i j}\right)\right. \\
\left.-\frac{\Lambda_{\pi}^{3}}{m_{\pi}^{3}} Y\left(\Lambda_{\pi} r_{i j}\right)\right]\left(\vec{\sigma}_{i} \cdot \vec{\sigma}_{j}\right) \sum_{a=1}^{3}\left(\lambda_{i}^{a} \cdot \lambda_{j}^{a}\right) \\
V_{\sigma}\left(\vec{r}_{i j}\right)=-\frac{g_{c h}^{2}}{4 \pi} \frac{\Lambda_{\sigma}^{2}}{\Lambda_{\sigma}^{2}-m_{\sigma}^{2}} m_{\sigma}\left[Y\left(m_{\sigma} r_{i j}\right)\right. \\
\left.\quad-\frac{\Lambda_{\sigma}}{m_{\sigma}} Y\left(\Lambda_{\sigma} r_{i j}\right)\right] \\
V_{K}\left(\vec{r}_{i j}\right)=\frac{g_{c h}^{2}}{4 \pi} \frac{m_{K}^{2}}{12 m_{i} m_{j}} \frac{\Lambda_{K}^{2}}{\Lambda_{K}^{2}-m_{K}^{2}} m_{K}\left[Y\left(m_{K} r_{i j}\right)\right. \\
\left.-\frac{\Lambda_{K}^{3}}{m_{K}^{3}} Y\left(\Lambda_{K} r_{i j}\right)\right]\left(\vec{\sigma}_{i} \cdot \vec{\sigma}_{j}\right) \sum_{a=4}^{7}\left(\lambda_{i}^{a} \cdot \lambda_{j}^{a}\right) \\
V_{\eta}\left(\vec{r}_{i j}\right)=\frac{g_{c h}^{2}}{4 \pi} \frac{m_{\eta}^{2}}{12 m_{i} m_{j}} \frac{\Lambda_{\eta}^{2}}{\Lambda_{\eta}^{2}-m_{\eta}^{2}} m_{\eta}\left[Y\left(m_{\eta} r_{i j}\right)\right. \\
\left.-\frac{\Lambda_{\eta}^{3}}{m_{\eta}^{3}} Y\left(\Lambda_{\eta} r_{i j}\right)\right]\left(\vec{\sigma}_{i} \cdot \vec{\sigma}_{j}\right)\left[\cos \theta_{p}\left(\lambda_{i}^{8} \cdot \lambda_{j}^{8}\right)-\sin \theta_{p}\right]
\end{gathered}
$$

where $Y(x)=e^{-x} / x$ is the standard Yukawa function. The physical $\eta$ meson, instead of the octet one, is considered by introducing the angle $\theta_{p}$. The $\lambda^{a}$ are the SU(3) flavor GellMann matrices. Taken from their experimental values, $m_{\pi}$, $m_{K}$, and $m_{\eta}$ are the masses of the SU(3) Goldstone bosons. The value of $m_{\sigma}$ used herein is given by the partially conserved axial current relation $m_{\sigma}^{2} \simeq m_{\pi}^{2}+4 m_{u, d}^{2}$ [63]. Note, however, that better determinations of the mass of the $\sigma$ meson have been reported since then [64,65] — see also the recent review [66]; one should simply consider the value used here as a model parameter. Finally, the chiral coupling constant $g_{c h}$ is determined from the $\pi N N$ coupling constant through

$$
\frac{g_{c h}^{2}}{4 \pi}=\frac{9}{25} \frac{g_{\pi N N}^{2}}{4 \pi} \frac{m_{u, d}^{2}}{m_{N}^{2}}
$$

which assumes that flavor $\mathrm{SU}(3)$ is an exact symmetry broken only by the different mass of the strange quark. Herein, we should notice that only one $\bar{q}(q=u, d)$ and one $s$ light quark are considered in the tetraquark system; hence, the $\pi$-meson exchange potential will be excluded in the chiral interaction.
Color confinement should be encoded in the nonAbelian character of QCD. It has been demonstrated by lattice-regularized QCD that multigluon exchanges produce an attractive linearly rising potential proportional to the distance between infinite-heavy quarks [67]. However, the spontaneous creation of light-quark pairs from the QCD vacuum may give rise at the same scale to a breakup of the created color flux tube [67]. These two observations can be described phenomenologically by

$$
V_{\mathrm{CON}}\left(\vec{r}_{i j}\right)=\left[-a_{c}\left(1-e^{-\mu_{c} r_{i j}}\right)+\Delta\right]\left(\lambda_{i}^{c} \cdot \lambda_{j}^{c}\right),
$$

where $a_{c}, \mu_{c}$, and $\Delta$ are model parameters, ${ }^{1}$ and the SU(3) color Gell-Mann matrices are denoted as $\lambda^{c}$. One can see in Eq. (10) that the potential is linear at short interquark distances with an effective confinement strength $\sigma=-a_{c} \mu_{c}\left(\lambda_{i}^{c} \cdot \lambda_{j}^{c}\right)$, while it becomes constant at large distances, $V_{\mathrm{thr}}=\left(\Delta-a_{c}\right)\left(\lambda_{i}^{c} \cdot \lambda_{j}^{c}\right)$.

Beyond the chiral symmetry-breaking scale, one expects the dynamics to be governed by QCD perturbative effects. In particular, the one-gluon exchange potential (which includes the so-called Coulomb and color-magnetic interactions) is the leading-order contribution:

$$
\begin{aligned}
V_{\mathrm{OGE}}\left(\vec{r}_{i j}\right)= & \frac{1}{4} \alpha_{s}\left(\lambda_{i}^{c} \cdot \lambda_{j}^{c}\right)\left[\frac{1}{r_{i j}}\right. \\
& \left.-\frac{1}{6 m_{i} m_{j}}\left(\vec{\sigma}_{i} \cdot \vec{\sigma}_{j}\right) \frac{e^{-r_{i j} / r_{0}\left(\mu_{i j}\right)}}{r_{i j} r_{0}^{2}\left(\mu_{i j}\right)}\right],
\end{aligned}
$$

where $r_{0}\left(\mu_{i j}\right)=\hat{r}_{0} / \mu_{i j}$ is a regulator which depends on the reduced mass of the $q \bar{q}$ pair, the Pauli matrices are denoted by $\vec{\sigma}$, and the contact term has been regularized as

$$
\delta\left(\vec{r}_{i j}\right) \sim \frac{1}{4 \pi r_{0}^{2}\left(\mu_{i j}\right)} \frac{e^{-r_{i j} / r_{0}\left(\mu_{i j}\right)}}{r_{i j}} .
$$

An effective scale-dependent strong coupling constant, $\alpha_{s}\left(\mu_{i j}\right)$, provides a consistent description of mesons and baryons from light to heavy quark sectors. We use the definition of Ref. [47]:

$$
\alpha_{s}\left(\mu_{i j}\right)=\frac{\alpha_{0}}{\ln \left(\frac{\mu_{i j}^{2} \mu_{0}^{2}}{\Lambda_{0}^{2}}\right)},
$$

in which $\alpha_{0}, \mu_{0}$, and $\Lambda_{0}$ are parameters of the model.

The model parameters are listed in Table I. Additionally, for later concern, Table II lists theoretical and experimental (if available) masses of $1 S$ and $2 S$ states of $K^{(*)}, D^{(*)}, D_{s}^{(*)}$,

\footnotetext{
${ }^{1}$ It is widely believed that confinement is flavor independent, and, thus, it should be constrained by the light hadron spectra despite our aim to determine energy states in heavier quark sectors $[46,68]$.
} 
TABLE I. Model parameters.

\begin{tabular}{llc}
\hline \hline Quark masses & $m_{q}(q=u, d)(\mathrm{MeV})$ & 313 \\
& $m_{s}(\mathrm{MeV})$ & 555 \\
Goldstone bosons & $m_{c}(\mathrm{MeV})$ & 1752 \\
& $\Lambda_{\sigma}\left(\mathrm{fm}^{-1}\right)$ & 4.20 \\
& $\Lambda_{\eta}=\Lambda_{K}\left(\mathrm{fm}^{-1}\right)$ & 5.20 \\
& $g_{c h}^{2} /(4 \pi)$ & 0.54 \\
Confinement & $\theta_{P}\left({ }^{\circ}\right)$ & -15 \\
& $a_{c}(\mathrm{MeV})$ & 430 \\
& $\mu_{c}\left(\mathrm{fm}^{-1}\right)$ & 0.70 \\
OGE & $\Delta\left(\mathrm{MeV}^{2}\right)$ & 181.10 \\
& $\alpha_{0}$ & 2.118 \\
& $\Lambda_{0}(\mathrm{fm}-1)$ & 0.113 \\
& $\mu_{0}(\mathrm{MeV})$ & 36.976 \\
& $\hat{r}_{0}(\mathrm{MeV} \mathrm{fm})$ & 28.170 \\
\hline \hline
\end{tabular}

TABLE II. Theoretical and experimental (if available) masses of $n L=1 S$ and $2 S$ states of $K^{(*)}, D^{(*)}, D_{s}^{(*)}, \eta_{c}$, and $J / \psi$ mesons.

\begin{tabular}{lccc}
\hline \hline Meson & $n L$ & $M_{\text {The }}(\mathrm{MeV})$ & $M_{\text {Exp }}(\mathrm{MeV})$ \\
\hline$K$ & $1 S$ & 481 & 494 \\
& $2 S$ & 1468 & $\ldots$ \\
$K^{*}$ & $1 S$ & 907 & 892 \\
& $2 S$ & 1621 & $\ldots$ \\
$D$ & $1 S$ & 1897 & 1870 \\
& $2 S$ & 2648 & $\ldots$ \\
$D^{*}$ & $1 S$ & 2017 & 2007 \\
& $2 S$ & 2704 & $\ldots$ \\
$D_{s}$ & $1 S$ & 1989 & $\ldots 68$ \\
& $2 S$ & 2705 & 2112 \\
$D_{s}^{*}$ & $1 S$ & 2115 & $\ldots$ \\
& $2 S$ & 2769 & $\ldots$ \\
$\eta_{c}$ & $1 S$ & 2989 & 3097 \\
$J / \psi$ & $2 S$ & 3627 & $\ldots$ \\
$\psi$ & $1 S$ & 3097 & \\
\hline \hline
\end{tabular}

$\eta_{c}$, and $J / \psi$ mesons predicted within our theoretical framework.

Figure 1 shows seven kinds of configurations for the $c \bar{c} s \bar{q}$ tetraquark system. In particular, Figs. 1(a) and 1(b) are meson-meson structures, Fig. 1(c) is the diquarkantidiquark one, and Figs. 1(d)-1(g) show the remaining multibody rearrangement channels in which the quarks (antiquarks) are coupled in an established sequence with no repetition; they are usually called K-type configurations. All of them, and their couplings, are considered in our investigation. However, for the purpose of solving a manageable four-body problem, the K-type configurations are sometimes restricted. It is important to note herein that just one configuration would be enough for the calculation, if all radial and orbital excited states were taken into account; however, this is obviously much less

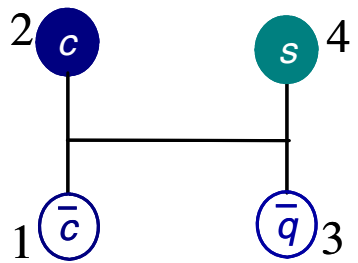

(a)

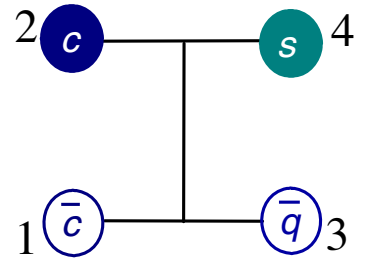

(c)

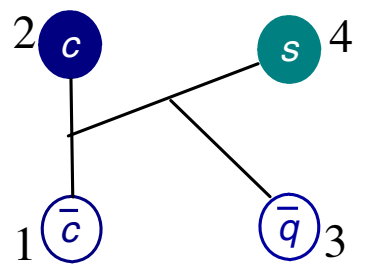

(e)

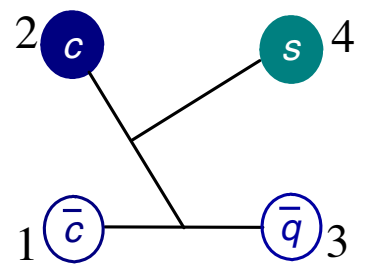

(g)

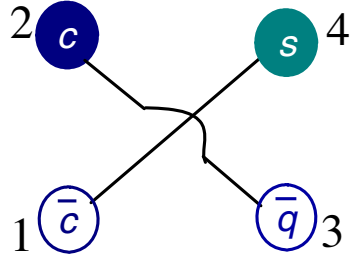

(b)

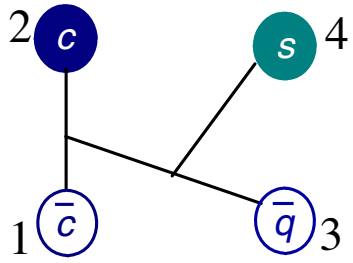

(d)

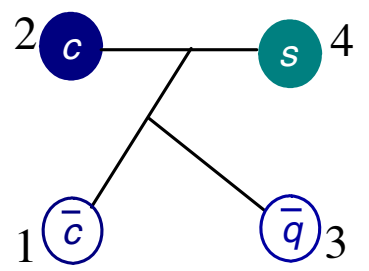

(f)
FIG. 1. Seven types of configurations in $c \bar{c} s \bar{q}(q=u, d)$ tetraquarks. (a) and (b) are meson-meson structures, (c) is a diquark-antidiquark one, and the other K-type structures are from (d) to $(\mathrm{g})$.

efficient, and, thus, an economic way is to combine the different configurations in the ground state to perform the calculation.

The multiquark system's wave function at the quark level is an internal product of color, spin, flavor, and space terms. Concerning the color degree of freedom, the colorless wave function of a four-quark system in mesonmeson configuration, as illustrated in Figs. 1(a) and 1(b), can be obtained by either two coupled color-singlet clusters, $1 \otimes 1$ :

$$
\chi_{1}^{c}=\frac{1}{3}(\bar{r} r+\bar{g} g+\bar{b} b) \times(\bar{r} r+\bar{g} g+\bar{b} b),
$$

or two coupled color-octet clusters, $8 \otimes 8$ : 


$$
\begin{aligned}
\chi_{2}^{c}= & \frac{\sqrt{2}}{12}(3 \bar{b} r \bar{r} b+3 \bar{g} r \bar{r} g+3 \bar{b} g \bar{g} b+3 \bar{g} b \bar{b} g+3 \bar{r} g \bar{g} r \\
& +3 \bar{r} b \bar{b} r+2 \bar{r} r \bar{r} r+2 \bar{g} g \bar{g} g+2 \bar{b} b \bar{b} b-\bar{r} r \bar{g} g \\
& -\bar{g} g \bar{r} r-\bar{b} b \bar{g} g-\bar{b} b \bar{r} r-\bar{g} g \bar{b} b-\bar{r} r \bar{b} b) .
\end{aligned}
$$

These two color states are the so-called color-singlet and hidden-color channels, respectively.

The color wave functions associated to the diquarkantidiquark structure shown in Fig. 1(c) are the coupled color triplet-antitriplet clusters, $3 \otimes \overline{3}$ :

$$
\begin{aligned}
\chi_{3}^{c}= & \frac{\sqrt{3}}{6}(\bar{r} r \bar{g} g-\bar{g} r \bar{r} g+\bar{g} g \bar{r} r-\bar{r} g \bar{g} r+\bar{r} r \bar{b} b \\
& -\bar{b} r \bar{r} b+\bar{b} b \bar{r} r-\bar{r} b \bar{b} r+\bar{g} g \bar{b} b-\bar{b} g \bar{g} b \\
& +\bar{b} b \bar{g} g-\bar{g} b \bar{b} g),
\end{aligned}
$$

and the coupled color sextet-antisextet clusters, $6 \otimes \overline{6}$ :

$$
\begin{aligned}
\chi_{4}^{c}= & \frac{\sqrt{6}}{12}(2 \bar{r} r \bar{r} r+2 \bar{g} g \bar{g} g+2 \bar{b} b \bar{b} b+\bar{r} r \bar{g} g+\bar{g} r \bar{r} g \\
& +\bar{g} g \bar{r} r+\bar{r} g \bar{g} r+\bar{r} r \bar{b} b+\bar{b} r \bar{r} b+\bar{b} b \bar{r} r \\
& +\bar{r} b \bar{b} r+\bar{g} g \bar{b} b+\bar{b} g \bar{g} b+\bar{b} b \bar{g} g+\bar{g} b \bar{b} g) .
\end{aligned}
$$

Meanwhile, the colorless wave functions of the K-type structures shown in Figs. 1(d)-1(g) are

$$
\begin{aligned}
\chi_{5}^{c}= & \frac{1}{6 \sqrt{2}}(\bar{r} r \bar{r} r+\bar{g} g \bar{g} g-2 \bar{b} b \bar{b} b)+\frac{1}{2 \sqrt{2}} \\
& \times(\bar{r} b \bar{b} r+\bar{r} g \bar{g} r+\bar{g} b \bar{b} g+\bar{g} r \bar{r} g+\bar{b} g \bar{g} b+\bar{b} r \bar{r} b) \\
& -\frac{1}{3 \sqrt{2}}(\bar{g} g \bar{r} r+\bar{r} r \bar{g} g)+\frac{1}{6 \sqrt{2}} \\
& \times(\bar{b} b \bar{r} r+\bar{b} b \bar{g} g+\bar{r} r \bar{b} b+\bar{g} g \bar{b} b), \\
& \chi_{6}^{c}=\chi_{1}^{c}, \\
\chi_{7}^{c}=\chi_{1}^{c}, & \frac{1}{4}\left(1-\frac{1}{\sqrt{6}}\right) \bar{r} r \bar{g} g-\frac{1}{4}\left(1+\frac{1}{\sqrt{6}}\right) \bar{g} g \bar{g} g-\frac{1}{4 \sqrt{3}} \bar{r} g \bar{g} r \\
& +\frac{1}{2 \sqrt{2}}(\bar{r} b \bar{b} r+\bar{g} b \bar{b} g+\bar{b} g \bar{g} b+\bar{g} r \bar{r} g+\bar{b} r \bar{r} b) \\
& +\frac{1}{2 \sqrt{6}}(\bar{r} r \bar{b} b-\bar{g} g \bar{b} b+\bar{b} b \bar{g} g+\bar{g} g \bar{r} r-\bar{b} b \bar{r} r), \quad(2 \\
\chi_{9}^{c}= & \frac{1}{2 \sqrt{6}}(\bar{r} b \bar{b} r+\bar{r} r \bar{b} b+\bar{g} b \bar{b} g+\bar{g} g \bar{b} b+\bar{r} g \bar{g} r+\bar{r} r \bar{g} g \\
& +\bar{b} b \bar{g} g+\bar{b} g \bar{g} b+\bar{g} g \bar{r} r+\bar{g} r \bar{r} g+\bar{b} b \bar{r} r+\bar{b} r \bar{r} b) \\
& +\frac{1}{\sqrt{6}}(\bar{r} r \bar{r} r+\bar{g} g \bar{g} g+\bar{b} b \bar{b} b),
\end{aligned}
$$

$$
\begin{aligned}
\chi_{10}^{c}= & \frac{1}{2 \sqrt{3}}(\bar{r} b \bar{b} r-\bar{r} r \bar{b} b+\bar{g} b \bar{b} g-\bar{g} g \bar{b} b+\bar{r} g \bar{g} r-\bar{r} r \bar{g} g \\
& -\bar{b} b \bar{g} g+\bar{b} g \bar{g} b-\bar{g} g \bar{r} r+\bar{g} r \bar{r} g-\bar{b} b \bar{r} r+\bar{b} r \bar{r} b),
\end{aligned}
$$

$$
\begin{aligned}
& \chi_{11}^{c}=\chi_{9}^{c}, \\
& \chi_{12}^{c}=-\chi_{10}^{c} .
\end{aligned}
$$

As for the flavor degree of freedom, since the quark content of the investigated tetraquark system is $c \bar{c} s \bar{q}$ $(q=u, d)$, only the $I=1 / 2$ sector is discussed. The flavor wave function is denoted as $\chi_{I, M_{I}}^{f}$, where the third component of the isospin, $M_{I}$, is fixed to be equal to $I$ for simplicity, since the Hamiltonian does not have a flavordependent interaction which can distinguish the third component of the isospin quantum number.

We are going to considered $S$-wave ground states with spin ranging from $S=0$ to 2 . Therefore, the spin wave functions $\chi_{S, M_{S}}^{\sigma_{i}}$ are given by $\left(M_{S}\right.$ can be set to be equal to $S$ without loss of generality)

$\chi_{0,0}^{\sigma_{u_{1}}}(4)=\chi_{00}^{\sigma} \chi_{00}^{\sigma}$,

$\chi_{0,0}^{\sigma_{u_{2}}}(4)=\frac{1}{\sqrt{3}}\left(\chi_{11}^{\sigma} \chi_{1,-1}^{\sigma}-\chi_{10}^{\sigma} \chi_{10}^{\sigma}+\chi_{1,-1}^{\sigma} \chi_{11}^{\sigma}\right)$,

$$
\begin{aligned}
\chi_{0,0}^{\sigma_{u_{3}}}(4)= & \frac{1}{\sqrt{2}}\left(\left(\sqrt{\frac{2}{3}} \chi_{11}^{\sigma} \chi_{\frac{1}{2},-\frac{1}{2}}^{\sigma}-\sqrt{\frac{1}{3}} \chi_{10}^{\sigma} \chi_{\frac{1}{2}, \frac{1}{2}}^{\sigma}\right) \chi_{\frac{1}{2},-\frac{1}{2}}^{\sigma}\right. \\
& \left.-\left(\sqrt{\frac{1}{3}} \chi_{10}^{\sigma} \chi_{\frac{1}{2},-\frac{1}{2}}^{\sigma}-\sqrt{\frac{2}{3}} \chi_{1,-1}^{\sigma} \chi_{\frac{1}{2}, \frac{1}{2}}^{\sigma}\right) \chi_{\frac{1}{2}, \frac{1}{2}}^{\sigma}\right),
\end{aligned}
$$

$\chi_{0,0}^{\sigma_{u_{4}}}(4)=\frac{1}{\sqrt{2}}\left(\chi_{00}^{\sigma} \chi_{\frac{1}{2}, 2}^{\sigma} \chi_{\frac{1}{2},-\frac{1}{2}}^{\sigma}-\chi_{00}^{\sigma} \chi_{\frac{1}{2}, \frac{1}{2}}^{\sigma} \chi_{\frac{1}{2}, \frac{1}{2}}^{\sigma}\right)$,

$\chi_{1,1}^{\sigma_{w_{1}}}(4)=\chi_{00}^{\sigma} \chi_{11}^{\sigma}$,

$\chi_{1,1}^{\sigma_{w_{2}}}(4)=\chi_{11}^{\sigma} \chi_{00}^{\sigma}$,

$\chi_{1,1}^{\sigma_{w_{3}}}(4)=\frac{1}{\sqrt{2}}\left(\chi_{11}^{\sigma} \chi_{10}^{\sigma}-\chi_{10}^{\sigma} \chi_{11}^{\sigma}\right)$,

$\chi_{1,1}^{\sigma_{w_{4}}}(4)=\sqrt{\frac{3}{4}} \chi_{11}^{\sigma} \chi_{\frac{1}{2}, \frac{1}{2}}^{\sigma} \chi_{\frac{1}{2},-\frac{1}{2}}^{\sigma}-\sqrt{\frac{1}{12}} \chi_{11}^{\sigma} \chi_{\frac{1}{2},-\frac{1}{2}}^{\sigma} \chi_{\frac{1}{2}, \frac{1}{2}}^{\sigma}$

$$
-\sqrt{\frac{1}{6}} \chi_{10}^{\sigma} \chi_{\frac{1}{2}, 2}^{\sigma} \chi_{\frac{1}{2}, \frac{1}{2}}^{\sigma},
$$

$\chi_{1,1}^{\omega_{w_{5}}}(4)=\left(\sqrt{\frac{2}{3}} \chi_{11}^{\sigma} \chi_{\frac{1}{2},-\frac{1}{2}}^{\sigma}-\sqrt{\frac{1}{3}} \chi_{10}^{\sigma} \chi_{\frac{1}{2}, \frac{1}{2}}^{\sigma}\right) \chi_{\frac{1}{2}, \frac{1}{2}}^{\sigma}$,

$\chi_{1,1}^{\sigma_{w_{6}}}(4)=\chi_{00}^{\sigma} \chi_{\frac{1}{2}, \frac{1}{2}}^{\sigma} \chi_{\frac{1}{2}, \frac{1}{2}}^{\sigma}$, 
TABLE III. The values of the superscripts $u_{1}, \ldots, u_{4}$ and $w_{1}, \ldots, w_{6}$ that determine the spin wave function for each configuration of the $c \bar{c} s \bar{q}$ tetraquark system.

\begin{tabular}{ccccccc}
\hline \hline & Dimeson & Diquark-antidiquark & $K_{1}$ & $K_{2}$ & $K_{3}$ & $K_{4}$ \\
\hline$u_{1}$ & 1 & 3 & & & & \\
$u_{2}$ & 2 & 4 & & & & \\
$u_{3}$ & & & 5 & 7 & 9 & 11 \\
$u_{4}$ & & 4 & 6 & 8 & 10 & 12 \\
$w_{1}$ & 1 & 5 & & & & \\
$w_{2}$ & 2 & 6 & & & & \\
$w_{3}$ & 3 & & 7 & 10 & 13 & 16 \\
$w_{4}$ & & & 8 & 11 & 14 & 17 \\
$w_{5}$ & & & 9 & 12 & 15 & 18 \\
$w_{6}$ & & & & & \\
\hline \hline
\end{tabular}

$$
\chi_{2,2}^{\sigma_{1}}(4)=\chi_{11}^{\sigma} \chi_{11}^{\sigma} .
$$

The superscripts $u_{1}, \ldots, u_{4}$ and $w_{1}, \ldots, w_{6}$ determine the spin wave function for each configuration of the $c \bar{c} s \bar{q}$ tetraquark system; their specific values are shown in Table III. Furthermore, the expressions above are obtained by considering the coupling of two subclusters whose spin wave functions are given by trivial SU(2) algebra, and the necessary basis reads as

$$
\begin{aligned}
\chi_{11}^{\sigma} & =\chi_{\frac{1}{2}, \frac{1}{2}, \frac{1}{2}, \frac{1}{2}}^{\sigma}, \\
\chi_{1,-1}^{\sigma} & =\chi_{\frac{1}{2},-\frac{1}{2}}^{\sigma} \chi_{\frac{1}{2},-\frac{1}{2}}^{\sigma}, \\
\chi_{10}^{\sigma} & =\frac{1}{\sqrt{2}}\left(\chi_{\frac{1}{2}, \frac{2}{2}}^{\sigma} \chi_{\frac{1}{2},-\frac{1}{2}}^{\sigma}+\chi_{\frac{1}{2},-\frac{1}{2}}^{\sigma} \chi_{\frac{1}{2}, \frac{1}{2}}^{\sigma}\right), \\
\chi_{00}^{\sigma} & =\frac{1}{\sqrt{2}}\left(\chi_{\frac{1}{2}, \frac{1}{2}}^{\sigma} \chi_{\frac{1}{2},-\frac{1}{2}}^{\sigma}-\chi_{\frac{1}{2},-\frac{1}{2}, \frac{1}{2}, \frac{1}{2}}^{\sigma}\right) .
\end{aligned}
$$

Among the different methods to solve the Schrödinger-like four-body bound state equation, we use the Rayleigh-Ritz variational principle, which is one of the most extended tools to solve eigenvalue problems because of its simplicity and flexibility. Moreover, we use the complex-range method, and, thus, the spatial wave function is written as follows:

$\psi_{L M_{L}}(\theta)=\left[\left[\phi_{n_{1} l_{1}}\left(\vec{\rho} e^{i \theta}\right) \phi_{n_{2} l_{2}}\left(\vec{\lambda} e^{i \theta}\right)\right]_{l} \phi_{n_{3} l_{3}}\left(\vec{R} e^{i \theta}\right)\right]_{L M_{L}}$,

where the internal Jacobi coordinates are defined as

$$
\begin{aligned}
& \vec{\rho}=\vec{x}_{1}-\vec{x}_{2(4)}, \\
& \vec{\lambda}=\vec{x}_{3}-\vec{x}_{4(2)},
\end{aligned}
$$

$$
\vec{R}=\frac{m_{1} \vec{x}_{1}+m_{2(4)} \vec{x}_{2(4)}}{m_{1}+m_{2(4)}}-\frac{m_{3} \vec{x}_{3}+m_{4(2)} \vec{x}_{4(2)}}{m_{3}+m_{4(2)}}
$$

for the meson-meson configurations of Figs. 1(a) and 1(b), where the numbers in parentheses are those corresponding to Fig. 1(b), and as

$$
\begin{aligned}
& \vec{\rho}=\vec{x}_{1}-\vec{x}_{3}, \\
& \vec{\lambda}=\vec{x}_{2}-\vec{x}_{4}, \\
& \vec{R}=\frac{m_{1} \vec{x}_{1}+m_{3} \vec{x}_{3}}{m_{1}+m_{3}}-\frac{m_{2} \vec{x}_{2}+m_{4} \vec{x}_{4}}{m_{2}+m_{4}}
\end{aligned}
$$

for the diquark-antidiquark structure in Fig. 1(c). The remaining K-type configurations shown in Figs. 1(d)-1(g) are $[i, j, k, l$ take values according to Figs. 1(d)-1(g)]

$$
\begin{aligned}
& \vec{\rho}=\vec{x}_{i}-\vec{x}_{j}, \\
& \vec{\lambda}=\vec{x}_{k}-\frac{m_{i} \vec{x}_{i}+m_{j} \vec{x}_{j}}{m_{i}+m_{j}}, \\
& \vec{R}=\vec{x}_{l}-\frac{m_{i} \vec{x}_{i}+m_{j} \vec{x}_{j}+m_{k} \vec{x}_{k}}{m_{i}+m_{j}+m_{k}} .
\end{aligned}
$$

It becomes obvious now that the center-of-mass kinetic term $T_{\text {c.m. }}$ can be completely eliminated for a nonrelativistic system defined in any of the above sets of relative coordinates.

A crucial aspect of the Rayleigh-Ritz variational method is the basis expansion of the trial wave function. We are going to use the GEM [59] in which each relative coordinate is expanded in terms of Gaussian basis functions whose sizes are taken in geometric progression. This method has proven to be very efficient on solving the bound-state problem of multiquark systems [36-41,58], and the details on how the geometric progression is fixed can be found in, e.g., Ref. [36]. Therefore, the form of the orbital wave functions $\phi$ 's in Eq. (41) is

$$
\phi_{n l m}\left(\vec{r} e^{i \theta}\right)=N_{n l}\left(r e^{i \theta}\right)^{l} e^{-\nu_{n}\left(r e^{i \theta}\right)^{2}} Y_{l m}(\hat{r}) .
$$

Since only $S$-wave states of charm(bottom)-strange tetraquarks are investigated in this work, no laborious Racah algebra is needed while computing matrix elements. In this case, the value of the spherical harmonic function is just a constant, viz. $Y_{00}=\sqrt{1 / 4 \pi}$.

Finally, the complete wave function that fulfills the Pauli principle is written as

$$
\Psi_{J M_{J}, I, i, j, k}(\theta)=\mathcal{A}\left[\left[\psi_{L}(\theta) \chi_{S}^{\sigma_{i}}(4)\right]_{J M_{J}} \chi_{I}^{f_{j}} \chi_{k}^{c}\right],
$$

where $\mathcal{A}$ is the antisymmetry operator of $c \bar{c} s \bar{q}$ tetraquark system and it just reads $\mathcal{A}=1$, since each of the four particles is nonidentical. 


\section{RESULTS}

In the present calculation, we investigate all possible $S$ wave hidden-charm tetraquarks with strangeness by taking into account dimeson, diquark-antidiquark, and K-type configurations. In our approach, a $c \bar{c} s \bar{q}$ tetraquark state has positive parity assuming that the angular momenta $l_{1}$, $l_{2}$, and $l_{3}$ in Eq. (41) are all equal to zero. Accordingly, the total angular momentum $J$ coincides with the total spin $S$ and can take values 0,1 , and 2 . Besides, the value of isospin $I$ can only be $1 / 2$ considering the quark content of the $c \bar{c} s \bar{q}$ system.

Tables IV-VI list the allowed meson-meson, diquarkantidiquark, and K-type channels; they are indexed in the first column, particular combinations of spin $\left(\chi_{J}^{\sigma_{i}}\right)$, flavor $\left(\chi_{I}^{f_{j}}\right)$, and color $\left(\chi_{k}^{c}\right)$ wave functions are shown in the second column, and the last column reflects the specific physical channel.

TABLE IV. All possible channels for $J^{P}=0^{+} c \bar{c} s \bar{q}$ tetraquark system. The second column shows the necessary basis combination in spin $\left(\chi_{J}^{\sigma_{i}}\right)$, flavor $\left(\chi_{I}^{f_{j}}\right)$, and color $\left(\chi_{k}^{c}\right)$ degrees of freedom. Particularly, the flavor index $(j) 1$ is of $\bar{c} c \bar{q} s$ and 2 is of $\bar{c} s \bar{q} c$, respectively. The superscript 1 and 8 stands for the color-singlet and hidden-color configurations of physical channels.

\begin{tabular}{lcc}
\hline \hline & $\chi_{J}^{\sigma_{i}} ; \chi_{I}^{f_{j}} ; \chi_{k}^{c}$ & \\
\cline { 2 - 3 } Index & {$[i ; j ; k]$} & Channel \\
\hline 1 & {$[1 ; 1 ; 1]$} & $\left(\eta_{c} K\right)^{1}$ \\
2 & {$[2 ; 1 ; 1]$} & $\left(J / \psi K^{*}\right)^{1}$ \\
3 & {$[1 ; 2 ; 1]$} & $\left(D D_{s}\right)^{1}$ \\
4 & {$[2 ; 2 ; 1]$} & $\left(D^{*} D_{s}^{*}\right)^{1}$ \\
5 & {$[1 ; 1 ; 2]$} & $\left(\eta_{c} K\right)^{8}$ \\
6 & {$[2 ; 1 ; 2]$} & $\left(J / \psi K^{*}\right)^{8}$ \\
7 & {$[1 ; 2 ; 2]$} & $\left(D D_{s}\right)^{8}$ \\
8 & {$[2 ; 2 ; 2]$} & $\left(D^{*} D_{s}^{*}\right)^{8}$ \\
9 & {$[3 ; 1 ; 3]$} & $(c s)_{3}(\bar{c} \bar{q})$ \\
10 & {$[4 ; 1 ; 3]$} & $(c s)_{3}^{*}(\bar{c} \bar{q})^{*}$ \\
11 & {$[3 ; 1 ; 4]$} & $(c s)_{6}(\bar{c} \bar{q})^{6}$ \\
12 & {$[4 ; 1 ; 4]$} & $(c s)_{6}^{*}(\bar{c} \bar{q})^{*}$ \\
13 & {$[5 ; 1 ; 5]$} & $K_{1}$ \\
14 & {$[6 ; 1 ; 5]$} & $K_{1}$ \\
15 & {$[5 ; 1 ; 6]$} & $K_{1}$ \\
16 & {$[6 ; 1 ; 6]$} & $K_{1}$ \\
17 & {$[7 ; 1 ; 7]$} & $K_{2}$ \\
18 & {$[8 ; 1 ; 7]$} & $K_{2}$ \\
19 & {$[7 ; 1 ; 8]$} & $K_{2}$ \\
20 & {$[8 ; 1 ; 8]$} & $K_{2}$ \\
21 & {$[9 ; 1 ; 9]$} & $K_{3}$ \\
22 & {$[10 ; 1 ; 9]$} & $K_{3}$ \\
23 & {$[9 ; 1 ; 10]$} & $K_{3}$ \\
24 & {$[10 ; 1 ; 10]$} & $K_{3}$ \\
25 & {$[11 ; 1 ; 11]$} & $K_{4}$ \\
26 & {$[12 ; 1 ; 11]$} & $K_{4}$ \\
27 & {$[11 ; 1 ; 12]$} & $K_{4}$ \\
28 & {$[12 ; 1 ; 12]$} & $K_{4}$ \\
\hline \hline
\end{tabular}

TABLE V. All possible channels for $J^{P}=1^{+} c \bar{c} s \bar{q}$ tetraquark system. The second and fifth columns show the necessary basis combination in spin $\left(\chi_{J}^{\sigma_{i}}\right)$, flavor $\left(\chi_{I}^{f_{j}}\right)$, and color $\left(\chi_{k}^{c}\right)$ degrees of freedom. Particularly, the flavor indices $(j) 1$ is of $\bar{c} c \bar{q} s$ and 2 is of $\bar{c} s \bar{q} c$, respectively. The superscript 1 and 8 stands for the colorsinglet and hidden-color configurations of physical channels.

\begin{tabular}{|c|c|c|c|c|c|}
\hline & $\bar{\sigma}_{J_{i}} ; \chi_{I}^{f_{j}} ; \chi$ & & & $\overline{\chi_{J}^{\sigma_{i}} ; \chi_{I}^{f_{j}} ; \chi_{k}^{c}}$ & \\
\hline Index & {$[i ; j ; k]$} & Channel & Index & {$[i ; j ; k]$} & Channel \\
\hline 1 & {$[1 ; 1 ; 1]$} & $\left(\eta_{c} K^{*}\right)^{1}$ & 19 & {$[7 ; 1 ; 5]$} & $K_{1}$ \\
\hline 2 & {$[2 ; 1 ; 1]$} & $(J / \psi K)^{1}$ & 20 & {$[8 ; 1 ; 5]$} & $K_{1}$ \\
\hline 3 & {$[3 ; 1 ; 1]$} & $\left(J / \psi K^{*}\right)^{1}$ & 21 & {$[9 ; 1 ; 5]$} & $K_{1}$ \\
\hline 4 & {$[1 ; 2 ; 1]$} & $\left(D D^{*}\right)^{1}$ & 22 & {$[7 ; 1 ; 6]$} & $K_{1}$ \\
\hline 5 & {$[2 ; 2 ; 1]$} & $\left(D^{*} D_{s}\right)^{1}$ & 23 & {$[8 ; 1 ; 6]$} & $K_{1}$ \\
\hline 6 & {$[3 ; 2 ; 1]$} & $\left(D^{*} D_{s}^{*}\right)^{1}$ & 24 & {$[9 ; 1 ; 6]$} & $K_{1}$ \\
\hline 7 & {$[1 ; 1 ; 2]$} & $\left(\eta_{c} K^{*}\right)^{8}$ & 25 & {$[10 ; 1 ; 7]$} & $K_{2}$ \\
\hline 8 & {$[2 ; 1 ; 2]$} & $(J / \psi K)^{8}$ & 26 & {$[11 ; 1 ; 7]$} & $K_{2}$ \\
\hline 9 & {$[3 ; 1 ; 2]$} & $\left(J / \psi K^{*}\right)^{8}$ & 27 & {$[12 ; 1 ; 7]$} & $K_{2}$ \\
\hline 10 & {$[1 ; 2 ; 2]$} & $\left(D D_{s}^{*}\right)^{8}$ & 28 & {$[10 ; 1 ; 8]$} & $K_{2}$ \\
\hline 11 & {$[2 ; 2 ; 2]$} & $\left(D^{*} D_{s}\right)^{8}$ & 29 & {$[11 ; 1 ; 8]$} & $K_{2}$ \\
\hline 12 & {$[3 ; 2 ; 2]$} & $\left(D^{*} D_{s}^{*}\right)^{8}$ & 30 & {$[12 ; 1 ; 8]$} & $K_{2}$ \\
\hline 13 & {$[4 ; 1 ; 3]$} & $(c s)_{3}(\bar{c} \bar{q})_{3}^{*}$ & 31 & {$[13 ; 1 ; 9]$} & $K_{3}$ \\
\hline 14 & {$[5 ; 1 ; 3]$} & $(c s)_{3}^{*}(\bar{c} \bar{q})_{\overline{3}}$ & 32 & {$[14 ; 1 ; 9]$} & $K_{3}$ \\
\hline 15 & {$[6 ; 1 ; 3]$} & $(c s)_{3}^{*}(\bar{c} \bar{q})_{3}^{*}$ & 33 & {$[15 ; 1 ; 9]$} & $K_{3}$ \\
\hline 16 & {$[4 ; 1 ; 4]$} & $(c s)_{6}(\bar{c} \bar{q})^{\frac{3}{6}}$ & 34 & {$[13 ; 1 ; 10]$} & $K_{3}$ \\
\hline 17 & {$[5 ; 1 ; 4]$} & $(c s)_{6}^{*}(\bar{c} \bar{q})_{\overline{6}}$ & 35 & {$[14 ; 1 ; 10]$} & $K_{3}$ \\
\hline 18 & {$[6 ; 1 ; 4]$} & $(c s)_{6}^{*}(\bar{c} \bar{q})_{6}^{*}$ & 36 & {$[15 ; 1 ; 10]$} & $K_{3}$ \\
\hline & & & 37 & {$[16 ; 1 ; 11]$} & $K_{4}$ \\
\hline & & & 38 & {$[17 ; 1 ; 11]$} & $K_{4}$ \\
\hline & & & 39 & {$[18 ; 1 ; 11]$} & $K_{4}$ \\
\hline & & & 40 & {$[16 ; 1 ; 12]$} & $K_{4}$ \\
\hline & & & 41 & {$[17 ; 1 ; 12]$} & $K_{4}$ \\
\hline & & & 42 & {$[18 ; 1 ; 12]$} & $K_{4}$ \\
\hline
\end{tabular}

Let us proceed now to describe in detail our theoretical findings for each $J^{P}=0^{+}, 1^{+}$, and $2^{+}$sector of $c \bar{c} s \bar{q}$ tetraquarks. Three subsections are presented in the following, and, in order to explore the detailed nature of the found states, three kinds of calculations are performed, i.e., the $(c \bar{c})(s \bar{q})$ dimeson configuration along with diquark-antidiquark and K-type ones in a coupled-channel study, the $(c \bar{q})(s \bar{c})$ dimeson configuration coupled with the other two types of exotic structures, and a complete coupled-channel investigation.

\section{A. The $J^{P}=0^{+} c \bar{c} s \bar{q}$ tetraquark system}

Table VII shows our calculated results of the lowest-lying $c \bar{c} s \bar{q}$ tetraquark states in a real-range study. The allowed dimeson, diquark-antidiquark, and K-type configurations are listed in the first column; when possible, the experimental value of the noninteracting meson-meson threshold is labeled in parentheses. Each channel is assigned an index in the second column. The theoretical mass obtained in each channel is shown in the third column, and the coupledchannel result for each kind of configuration is presented in 
TABLE VI. All possible channels for $J^{P}=2^{+} c \bar{c} s \bar{q}$ tetraquark system. The second column shows the necessary basis combination in spin $\left(\chi_{J}^{\sigma_{i}}\right)$, flavor $\left(\chi_{I}^{f_{j}}\right)$, and color $\left(\chi_{k}^{c}\right)$ degrees of freedom. Particularly, the flavor indices $(j) 1$ is of $\bar{c} c \bar{q} s$ and 2 is of $\bar{c} s \bar{q} c$, respectively. The superscript 1 and 8 stands for the color-singlet and hidden-color configurations of physical channels.

\begin{tabular}{lcc}
\hline \hline & $\frac{\chi_{J}^{\sigma_{i}} ; \chi_{I}^{f_{j}} ; \chi_{k}^{c}}{}$ & \\
\cline { 2 - 3 } Index & {$[i ; j ; k]$} & Channel \\
\hline 1 & {$[1 ; 1 ; 1]$} & $\left(J / \psi K^{*}\right)^{1}$ \\
2 & {$[1 ; 2 ; 1]$} & $\left(D^{*} D_{s}^{*}\right)^{1}$ \\
3 & {$[1 ; 1 ; 2]$} & $\left(J / \psi K^{*}\right)^{8}$ \\
4 & {$[1 ; 2 ; 2]$} & $\left(D^{*} D_{s}^{*}\right)^{8}$ \\
5 & {$[1 ; 1 ; 3]$} & $(c s)_{3}^{*}(\bar{c} \bar{q})_{3}^{*}$ \\
6 & {$[1 ; 1 ; 4]$} & $(c s)_{6}^{*}(\bar{c} \bar{q})^{*}$ \\
7 & {$[1 ; 1 ; 5]$} & $K_{1}$ \\
8 & {$[1 ; 1 ; 6]$} & $K_{1}$ \\
9 & {$[1 ; 1 ; 7]$} & $K_{2}$ \\
10 & {$[1 ; 1 ; 8]$} & $K_{2}$ \\
11 & {$[1 ; 1 ; 9]$} & $K_{3}$ \\
12 & {$[1 ; 1 ; 10]$} & $K_{3}$ \\
13 & {$[1 ; 1 ; 11]$} & $K_{4}$ \\
14 & {$[1 ; 1 ; 12]$} & $K_{4}$ \\
\hline \hline
\end{tabular}

the last one. When a complete coupled-channel calculation is performed, the last row of the table indicates the lowest-lying mass. We show in Figs. 2-4 the distribution of complex eigenenergies when the CSM is used in the coupled-channel calculation, and, therein, the obtained resonance states are indicated inside circles. Furthermore, when all channels listed in Table IV are considered excluding the dimeson states in color-singlet configurations, the obtained resonances below $4.3 \mathrm{GeV}$ along with their inner structures are summarized in Table VIII.

Exotic states in $(c \bar{c})(s \bar{q})$ dimeson channels.-Table VII shows that the lowest channel of $c \bar{c} s \bar{q}$ tetraquark with spin-parity $0^{+}$is the color-singlet channel of $\eta_{c} K$ with a theoretical mass of $3470 \mathrm{MeV}$, which is just the theoretical threshold value, and, thus, bounding is not possible here. This fact also holds for another higher meson-meson channel, $J / \psi K^{*}$, whose calculated mass is $4004 \mathrm{MeV}$. Then, the hidden-color structures for $\eta_{c} K$ and $J / \psi K^{*}$ have masses 4422 and $4416 \mathrm{MeV}$, respectively; obviously, these are much deviated from the relevant experimental data.

A complex-range coupled-channel calculation is performed in a further step. Figure 2 presents the calculated results, in which the $(c \bar{c})(s \bar{q})$ dimeson, diquark-antidiquark, and K-type configurations are considered. In a mass gap from 3.4 to $4.7 \mathrm{GeV}$, the calculated complex energy dots of $\eta_{c}(1 S) K(1 S), J / \psi(1 S) K^{*}(1 S), \eta_{c}(2 S) K(1 S), \eta_{c}(1 S)$ $K(2 S)$, and $\psi(2 S) K^{*}(1 S)$ channels are generally well aligned along their corresponding threshold lines. In particular, with a complex angle varied from $0^{\circ}$ to $6^{\circ}$, these energy
TABLE VII. Lowest-lying $c \bar{c} s \bar{q}$ tetraquark states with $J^{P}=0^{+}$ calculated within the real range formulation of the chiral quark model. The allowed meson-meson, diquark-antidiquark, and Ktype configurations are listed in the first column; when possible, the experimental value of the noninteracting meson-meson threshold is labeled in parentheses. Each channel is assigned an index in the second column. The theoretical mass obtained in each channel is shown in the third column, and the coupled result for each kind of configuration is presented in the last column. When a complete coupled-channel calculation is performed, the last row of the table indicates the lowest-lying mass (unit, $\mathrm{MeV}$ ).

\begin{tabular}{|c|c|c|c|}
\hline Channel & Index & $M$ & Mixed \\
\hline$\left(\eta_{c} K\right)^{1}(3475)$ & 1 & 3470 & \\
\hline$\left(J / \psi K^{*}\right)^{1}(3989)$ & 2 & 4004 & \\
\hline$\left(D D_{s}\right)^{1}(3838)$ & 3 & 3886 & \\
\hline$\left(D^{*} D_{s}^{*}\right)^{1}(4119)$ & 4 & 4132 & 3470 \\
\hline$\left(\eta_{c} K\right)^{8}$ & 5 & 4422 & \\
\hline$\left(J / \psi K^{*}\right)^{8}$ & 6 & 4416 & \\
\hline$\left(D D_{s}\right)^{8}$ & 7 & 4398 & \\
\hline$\left(D^{*} D_{s}^{*}\right)^{8}$ & 8 & 4333 & 4177 \\
\hline$(c s)_{3}(\bar{c} \bar{q})_{\overline{3}}$ & 9 & 4320 & \\
\hline$(c s)_{3}^{*}(\bar{c} \bar{q})_{\frac{3}{3}}^{*}$ & 10 & 4334 & \\
\hline$(c s)_{6}(\bar{c} \bar{q})_{\overline{6}}^{3}$ & 11 & 4349 & \\
\hline$(c s)_{6}^{*}(\bar{c} \bar{q})_{\overline{6}}^{*}$ & 12 & 4224 & 4122 \\
\hline \multirow[t]{4}{*}{$K_{1}$} & 13 & 4407 & \\
\hline & 14 & 4420 & \\
\hline & 15 & 4296 & \\
\hline & 16 & 4080 & 4075 \\
\hline \multirow[t]{4}{*}{$K_{2}$} & 17 & 4283 & \\
\hline & 18 & 4062 & \\
\hline & 19 & 4407 & \\
\hline & 20 & 4419 & 4057 \\
\hline \multirow[t]{4}{*}{$K_{3}$} & 21 & 4217 & \\
\hline & 22 & 4353 & \\
\hline & 23 & 4320 & \\
\hline & 24 & 4319 & 4114 \\
\hline \multirow[t]{4}{*}{$K_{4}$} & 25 & 4241 & \\
\hline & 26 & 4372 & \\
\hline & 27 & 4326 & \\
\hline & 28 & 4324 & 4117 \\
\hline \multicolumn{3}{|c|}{ Complete coupled-channels: } & 3470 \\
\hline
\end{tabular}

poles are basically moving along the theoretical cut lines, and this fact confirms their nature as discrete-continuum states. In Fig. 2, one could find that three resonance poles exist, circled in green, whose masses and widths are $(4255,5.0)$, $(4567,1.6)$, and $(4675,1.9) \mathrm{MeV}$, respectively. These narrow resonances are obtained in the $(c \bar{c})(s \bar{q})$ dimeson channels along with the couplings in diquark-antidiquark and K-type configurations. Moreover, attending to their positions in the complex plane, the dominant components of them can be identified as $\eta_{c}(2 S) K(1 S)(4255), \eta_{c}(1 S) K(2 S)(4567)$, and $\psi(2 S) K^{*}(1 S)(4675)$, respectively. Accordingly, the reported $Z_{c s}(4220)$ and $X(4630)$ states can be related to the radial excitation of $\eta_{c} K$ and $\psi K^{*}$ in coupled-channel cases, without a $D^{(*)} D_{s}^{(*)}$ channel included. 


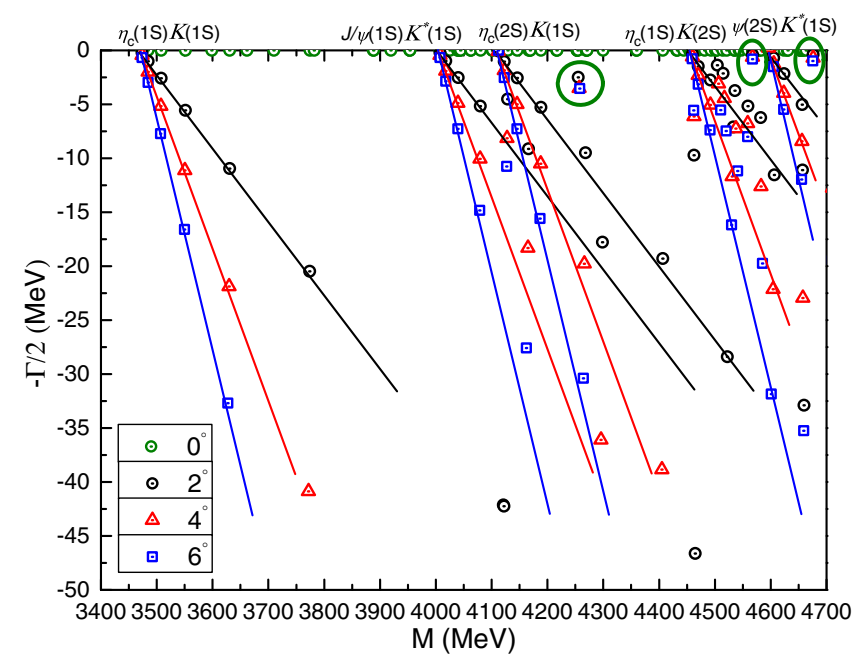

FIG. 2. The coupled-channel calculation of the $c \bar{c} s \bar{q}$ tetraquark system with $J^{P}=0^{+}$quantum numbers. Particularly, the $(c \bar{q})(s \bar{c})$ dimeson channels are excluded. We use the complexscaling method of the chiral quark model varying $\theta$ from $0^{\circ}$ to $6^{\circ}$.

Exotic states in $(c \bar{q})(s \bar{c})$ dimeson channels.-In a singlechannel calculation of $(c \bar{q})(s \bar{c})$ meson-meson configuration, the lowest mass $3886 \mathrm{MeV}$ is equal to the theoretical threshold value of $D D_{s}$ color-singlet channel in Table VII, and the other $(c \bar{q})(s \bar{c})$ dimeson channel, $D^{*} D_{s}^{*}$, is at $4132 \mathrm{MeV}$. Therefore, no bound state is found herein too, since the color-octet channels of $D D_{s}$ and $D^{*} D_{s}^{*}$ are both excited states at around $4.3 \mathrm{GeV}$.

In a further step, a coupled-channel calculation which includes the $(c \bar{q})(s \bar{c})$ dimeson, diquark-antidiquark, and K-type configurations is considered in the CSM. Figure 3 presents the general distributions of calculated complex energy dots. Particularly, in the top panel in Fig. 3, most of the complex poles of $D^{(*)} D_{s}^{(*)}$ are aligned well along the cut lines within a mass region from 3.8 to $4.7 \mathrm{GeV}$. However, there are dense distributions around 4.2 and $4.6 \mathrm{GeV}$, and so two enlarged panels are shown accordingly. First, two resonance poles are obtained in the middle panel in Fig. 3 whose mass gap ranges from 4.10 to $4.25 \mathrm{GeV}$. They can be identified as $D^{*}(1 S) D_{s}^{*}(1 S)$ narrow resonances with calculated masses and widths $(4150,2.2)$ and $(4185,8.2) \mathrm{MeV}$, respectively. For the lower resonance, despite its small decay width, our theoretical mass is compatible with the $Z_{c s}^{\prime}(4130)$ (tensor $D^{*} \bar{D}_{s}^{*}$ resonance) concluded by Refs. $[4,28]$.

The bottom panel in Fig. 3 shows the highest energy region, $4.55-4.70 \mathrm{GeV}$, where the radial excitations of $D D_{s}$ thresholds are clearly identified. Therein, the calculated complex dots are generally aligned along their corresponding threshold lines, and no stable resonance pole is found.

The fully coupled-channel case.-There are 28 channels under consideration for the $c \bar{c} s \bar{q}$ tetraquark with spinparity $0^{+}$. In Table VII, one could see that, apart from the
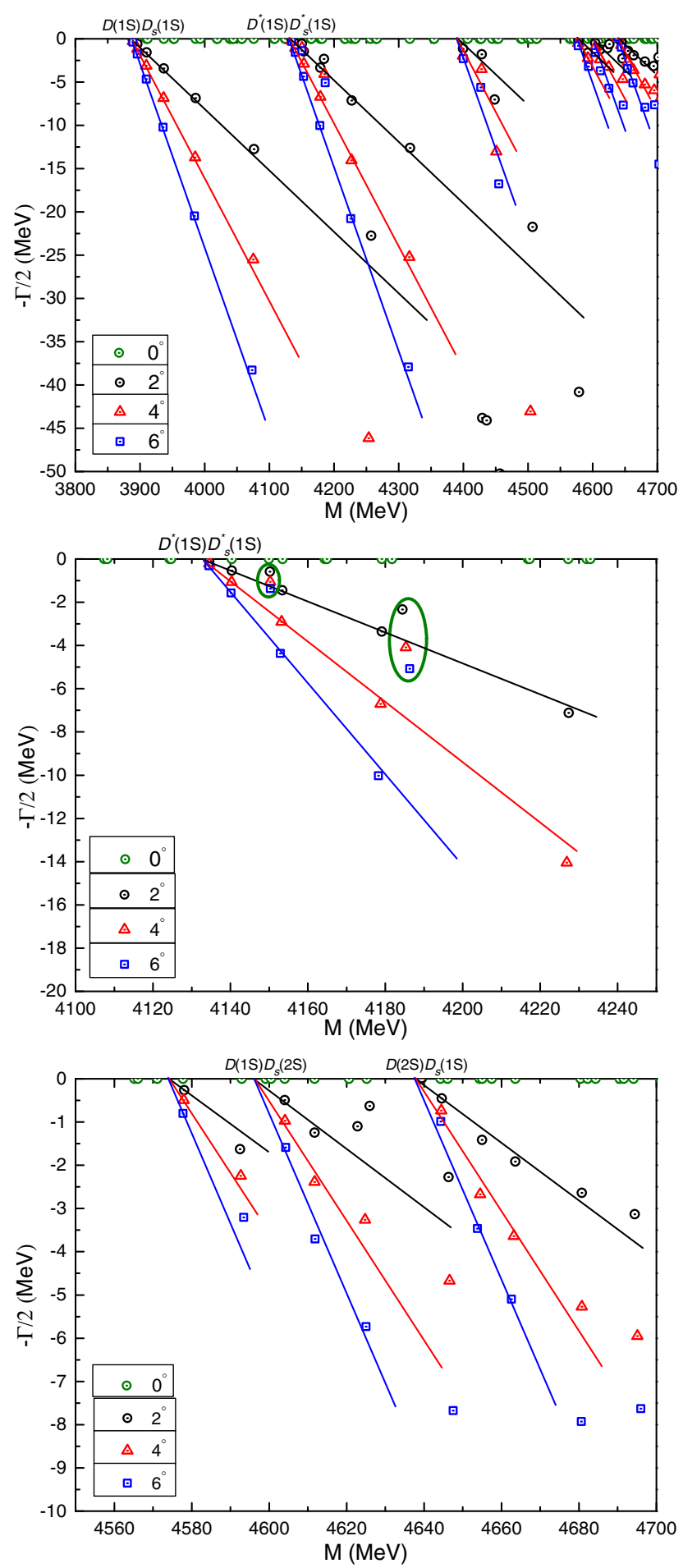

FIG. 3. Top panel: complex energy spectrum of the $c \bar{c} s \bar{q}$ system with $J^{P}=0^{+}$from the coupled-channel calculation with CSM. Particularly, the $(c \bar{c})(s \bar{q})$ dimeson channels are excluded. The parameter $\theta$ varies from $0^{\circ}$ to $6^{\circ}$. Middle panel: enlarged top panel, with real values of energy ranging from 4.10 to $4.25 \mathrm{GeV}$. Bottom panel: enlarged top panel, with real values of energy ranging from 4.55 to $4.70 \mathrm{GeV}$. 

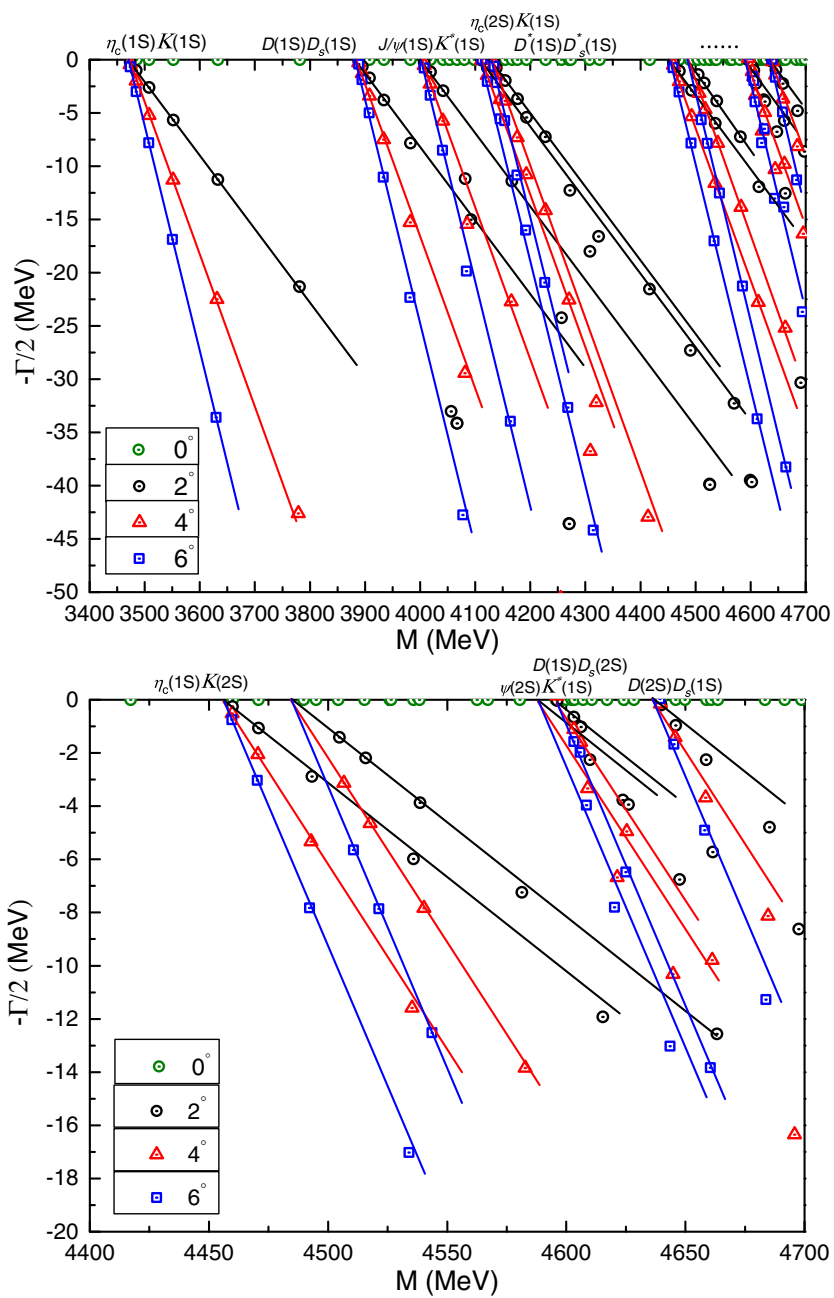

FIG. 4. Top panel: the complete coupled-channel calculation of the $c \bar{c} s \bar{q}$ tetraquark system with $J^{P}=0^{+}$quantum numbers. We use the complex-scaling method of the chiral quark model varying $\theta$ from $0^{\circ}$ to $6^{\circ}$. Bottom panel: enlarged top panel, with real values of energy ranging from 4.40 to $4.70 \mathrm{GeV}$.

dimeson channels in both color-singlet and color-octet cases, four allowed channels in diquark-antidiquark and K-type configurations are also considered. Generally, in each single-channel computation, masses of diquarkantidiquark structures are about $4.3 \mathrm{GeV}$, and $4.1-$ 4.4 GeV for K-type ones. Then, in coupled-channel calculations of each specific configurations, the lowest mass, $3470 \mathrm{MeV}$, is still just the theoretical threshold value of $\eta_{c} K$, and the other exotic structures are around $4.1 \mathrm{GeV}$. In particular, the coupled masses in $K_{1}$ and $K_{2}$ structures are both $\sim 4.05 \mathrm{GeV}$, which is close to the experimental mass of $Z_{c s}(4000)$. Finally, within the realrange investigation, the fully coupled-channel mass remains at $3470 \mathrm{MeV}$, which implies the coupling effect is quite weak and no bound state is available.

The exotic state within hidden-color channel could be a natural bound state; hence, a coupled-channel calculation of all those exotic structures which include color-octet, diquark-antidiquark, and K-type configurations is performed. Table VIII lists the obtained $c \bar{c} s \bar{q}$ tetraquark resonances below $4.3 \mathrm{GeV}$, and their inner structures are also investigated. The lowest resonance is at $3841 \mathrm{MeV}$, and the other four excited states are lying in 4.11$4.25 \mathrm{GeV}$. Apparently, these exotic resonance states are likely compact structures with interquark distance generally less than $1.1 \mathrm{fm}$, and the distance between $c \bar{c}$ is around $0.5 \mathrm{fm}$. They could be good candidates of exotic color structures in the strange hidden-charm tetraquark sector.

Additionally, the fully coupled-channel calculation is studied in a complex-range scaling. Figure 4 shows the distribution of our calculated complex energies. Particularly, in the top panel, the scattering states of $\eta_{c} K, J / \psi K^{*}, D D_{s}$, and $D^{*} D_{s}^{*}$ can be clearly identified within the mass region 3.4-4.7 GeV. Besides, since there is a dense distribution between 4.5 and $4.7 \mathrm{GeV}$, an enlarged part whose mass region is from 4.4 to $4.7 \mathrm{GeV}$ is shown in the bottom panel in Fig. 4. Therein, the scattering nature of the radial excited states $\eta_{c} K, \psi K^{*}$, and $D D_{s}$ is also well presented. One can realize that the obtained resonance states in the coupled-channel investigations discussed above, $\eta_{c}(2 S) K(1 S)(4255), \psi(2 S)$ $K^{*}(1 S)(4675), D^{*}(1 S) D_{s}^{*}(1 S)(4150)$, etc., turn to be scattering ones because, in a complete coupled-channel calculation, these resonances easily decay to lower $(c \bar{c})(s \bar{q})$ or $(c \bar{q})(s \bar{c})$ dimeson scattering states. This indicates a fact that the strange hidden-charm tetraquark resonances should be extremely harsh to be detected, and this finding is also related to the conclusions in Ref. [69].

TABLE VIII. The distance, in femtometers, between any two quarks of the $J^{P}=0^{+} c \bar{c} s \bar{q}$ tetraquark resonance state obtained in all exotic configurations' coupled-channel calculation. These resonances, which masses are below $4.3 \mathrm{GeV}$, are labeled in the first column.

\begin{tabular}{lcccccr}
\hline \hline State & $r_{\bar{c} c}$ & $r_{\bar{c} \bar{q}}$ & $r_{\bar{c} s}$ & $r_{c \bar{q}}$ & $r_{c s}$ & $r_{s \bar{q}}$ \\
\hline$Z_{c s}(3841)$ & 0.35 & 0.76 & 0.70 & 0.76 & 0.70 & 0.69 \\
$Z_{c s}(4105)$ & 0.51 & 0.84 & 0.69 & 0.77 & 0.76 & 0.85 \\
$Z_{c s}(4156)$ & 0.42 & 0.98 & 0.92 & 0.98 & 0.91 & 0.89 \\
$Z_{c s}(4193)$ & 0.42 & 1.16 & 1.13 & 1.15 & 1.14 & 1.02 \\
$Z_{c s}(4258)$ & 0.64 & 0.82 & 0.63 & 0.73 & 0.73 & 0.91 \\
\hline \hline
\end{tabular}




\section{B. The $J^{P}=1^{+} c \bar{c} s \bar{q}$ tetraquark system}

The available 42 channels that include meson-meson, diquark-antidiquark, and K-type structures are listed in Table V. The lowest-lying $c \bar{c} s \bar{q}$ tetraquark states with spinparity $J^{P}=1^{+}$are first calculated within the real-range approximation and summarized in Table IX. Particularly, the allowed channels are listed in the first and fifth columns, when possible, the noninteracting meson-meson experimental threshold values are labeled in parentheses. The assigned indexes for the channels are shown in the second and sixth columns. The theoretical mass obtained in each channel is shown in the third and seventh columns; besides, the coupled result for each kind of configuration is presented in the fourth and last columns. The last row in Table IX indicates the lowest-lying mass of the system in a fully coupled-channel case. When the CSM is used in the coupled-channel calculations, Figs. 5-7 show the distribution of complex eigenenergies and, therein, the obtained resonance states are indicated inside circles. Furthermore, when a calculation which all channels listed in Table $\mathrm{V}$ are considered except the color-singlet dimeson cases is performed, the obtained resonances within a mass region 3.9-4.2 GeV, along with their inner structures, are summarized in Table X. Now let us discuss the details of $c \bar{c} s \bar{q}$ tetraquark in each of the three kinds of investigation below.

Exotic states in $(c \bar{c})(s \bar{q})$ dimeson channels.-There are six $(c \bar{c})(s \bar{q})$ dimeson channels contributing to the tetraquark system in the $1^{+}$case, i.e., $\eta_{c} K^{*}, J / \psi K$, and $J / \psi K^{*}$ states in color-singlet and -octet channels. From Table IX, we find that the three color-singlet channels are all unbound, the lowest-lying one, $J / \psi K$, is at $3578 \mathrm{MeV}$, the next one is $\eta_{c} K^{*}$ with mass at $3896 \mathrm{MeV}$, and $J / \psi K^{*}$ is at $4004 \mathrm{MeV}$. Their corresponding hidden-color channels masses are all $\sim 4.4 \mathrm{GeV}$.

Additionally, a complex-range computation is performed in the coupled-channel study where the $(c \bar{c})(s \bar{q})$ dimeson structures, diquark-antidiquark configurations, and K-type ones are considered. Within a complex angle $\theta$ varied from $0^{\circ}$ to $6^{\circ}$, Fig. 5 shows the distribution of calculated complex energy dots. In particular, the scattering states of $J / \psi K$, $\eta_{c} K^{*}$, and $J / \psi K^{*}$ are well presented in a mass region 3.5$4.7 \mathrm{GeV}$ in the top panel in Fig. 5. However, there are three stable resonance poles, encircled by green lines, which can be identified as $\psi(2 S) K(1 S)$ molecular resonances. Their calculated masses and widths are, all in $\mathrm{MeV}$, $(4254,0.8)$, $(4267,6.2)$, and $(4303,1.0)$. As one could conclude, the $Z_{c s}(4220)$ would be explained, too, as a $\psi(2 S) K(1 S)$ (4254) resonance in the $1^{+}$state.

Meanwhile, since there is a dense distribution of complex energies at around $4.6 \mathrm{GeV}$, an enlarged panel whose mass range goes from 4.5 to $4.7 \mathrm{GeV}$ is shown at the bottom in Fig. 5. Therein, the radial excitation states of $\eta_{c} K^{*}$ and $J / \psi K^{(*)}$ are clearly presented, and no resonance pole is obtained.
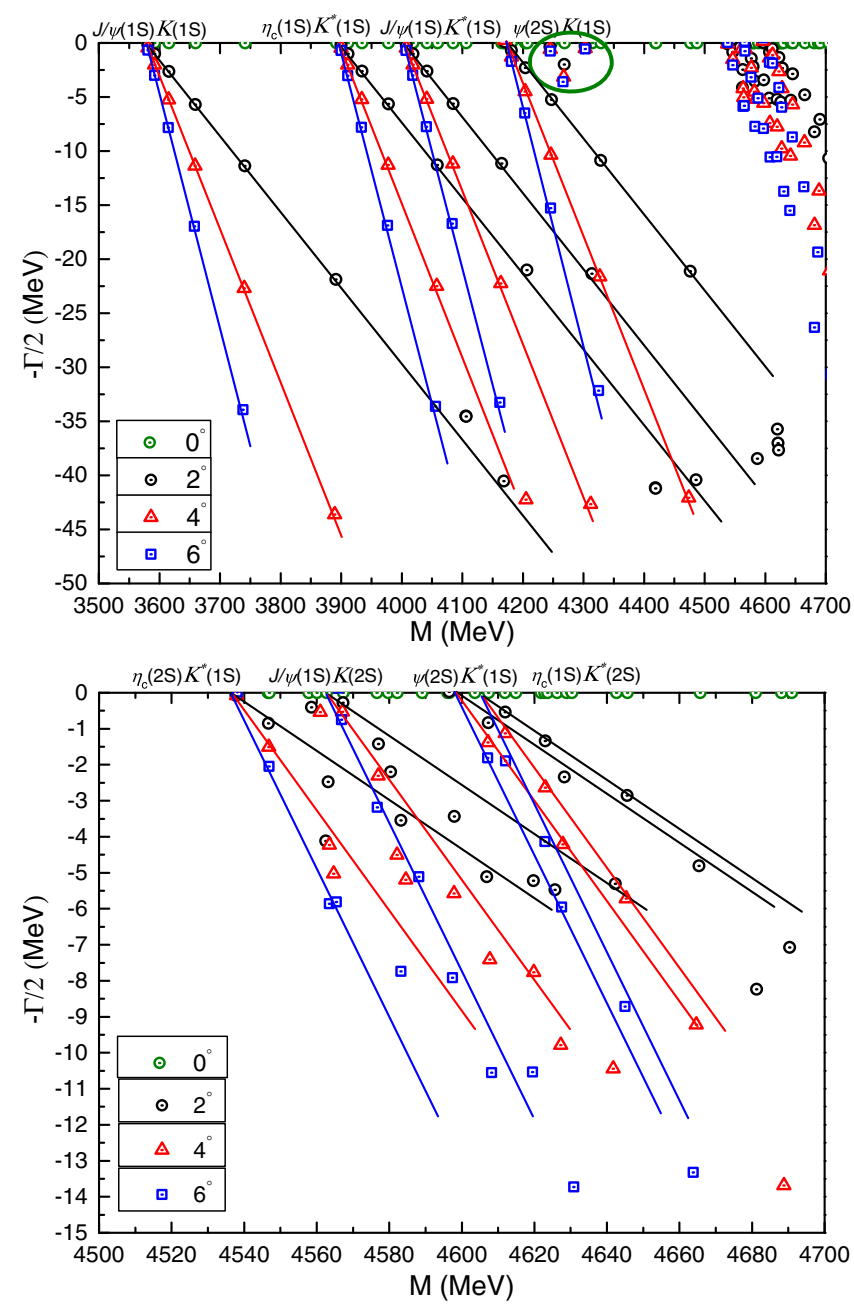

FIG. 5. Top panel: the coupled-channel calculation of the $c \bar{c} s \bar{q}$ tetraquark system with $J^{P}=1^{+}$quantum numbers. Particularly, the $(c \bar{q})(s \bar{c})$ dimeson channels are excluded. We use the complex-scaling method of the chiral quark model varying $\theta$ from $0^{\circ}$ to $6^{\circ}$. Bottom panel: enlarged top panel, with real values of energy ranging from 4.50 to $4.70 \mathrm{GeV}$.

Exotic states in $(c \bar{q})(s \bar{c})$ dimeson channels.-One can find in Table IX that there are six $(c \bar{q})(s \bar{c})$ dimeson channels which contribute to the tetraquark system with $1^{+}$quantum numbers, i.e., $D D_{s}^{*}, D^{*} D_{s}$, and $D^{*} D_{s}^{*}$ states in color-singlet and -octet channels. Within the real-range single-channel approach, their theoretical masses in colorsinglet channels are $\sim 4.0 \mathrm{GeV}$, except for $D^{*} D_{s}^{*}$ at $4132 \mathrm{MeV}$. The hidden-color channels masses are higher and generally lie at around $4.4 \mathrm{GeV}$. Although these values are quite close to the experimental data of $Z_{c s}(4000)$, the scattering nature of $D^{(*)} D_{s}^{(*)}$ channel remains.

The coupled-channel result by CSM is shown in Fig. 6, within a mass range from 4.0 to $4.7 \mathrm{GeV}$. The scattering nature of $D D_{s}^{*}$ and $D^{*} D_{s}$ is clearly demonstrated. Nevertheless, two narrow $D^{*}(1 S) D_{s}^{*}(1 S)$ resonances are obtained and circled in green. The calculated masses and 


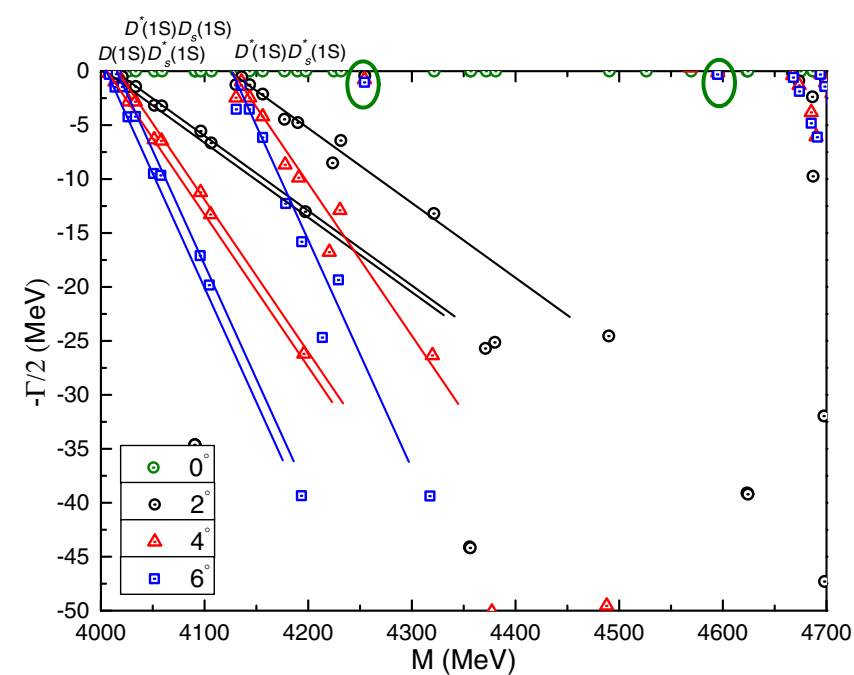

FIG. 6. The coupled-channel calculation of the $c \bar{c} s \bar{q}$ tetraquark system with $J^{P}=1^{+}$quantum numbers. Particularly, the $(c \bar{c})(s \bar{q})$ dimeson channels are excluded. We use the complexscaling method of the chiral quark model varying $\theta$ from $0^{\circ}$ to $6^{\circ}$.

widths are $(4254,1.6)$ and $(4594,0.6) \mathrm{MeV}$, respectively. Accordingly, there is a degeneration between the $D^{*}(1 S) D_{s}^{*}(1 S)$ and $\psi(2 S) K(1 S)$ channels at $4254 \mathrm{MeV}$.

The fully coupled-channel case.-Apart from the 12 meson-meson channels listed in Table IX, the single-channel calculation for diquark-antidiquark structures and K-type ones is also analyzed. In particular, there are six channels in each kind of configuration, the diquark-antidiquark channels are $\sim 4.3 \mathrm{GeV}$, and the K-type ones are lying from 4.2 to $4.4 \mathrm{GeV}$. In addition, when a coupled-channel study is done in each configuration, the lowest mass, $3578 \mathrm{MeV}$, is still equal to the theoretical threshold value of $J / \psi K$. The mass of the hidden-color coupled-channel result is higher and at $4224 \mathrm{MeV}$. Meanwhile, the diquark-antidiquark and K-type structure coupled masses are all around $4.18 \mathrm{GeV}$. However, no bound state is found in the $1^{+}$state when a complete coupled-channel calculation is studied, and the lowest mass remains at $3578 \mathrm{MeV}$.

In order to have a much more detailed investigation on the strange hidden-charm tetraquark in the $1^{+}$state, a coupled-channel computation of all exotic structures where only the dimeson structures in color-singlet channels are excluded is performed. Table $\mathrm{X}$ summarizes the $Z_{c s}$ resonances within 3.9-4.2 GeV. The five exotic states are compact structures, and the calculated distances between any two (anti)quark and quark-antiquark are less than $1.0 \mathrm{fm}$. Particularly, the two lower states, $Z_{c s}(3947)$ and $Z_{c s}(4038)$, are well compatible with the recently reported exotic structures [1,2]. Hence, the $Z_{c s}(3985)$ and $Z_{c s}(4000)$ should be considered as a $J^{P}=1^{+} c \bar{c} s \bar{q}$ tetraquark with a large compact component.

Last but not least, a fully coupled-channel calculation in CSM is investigated, and the result is listed in Fig. 7. First,
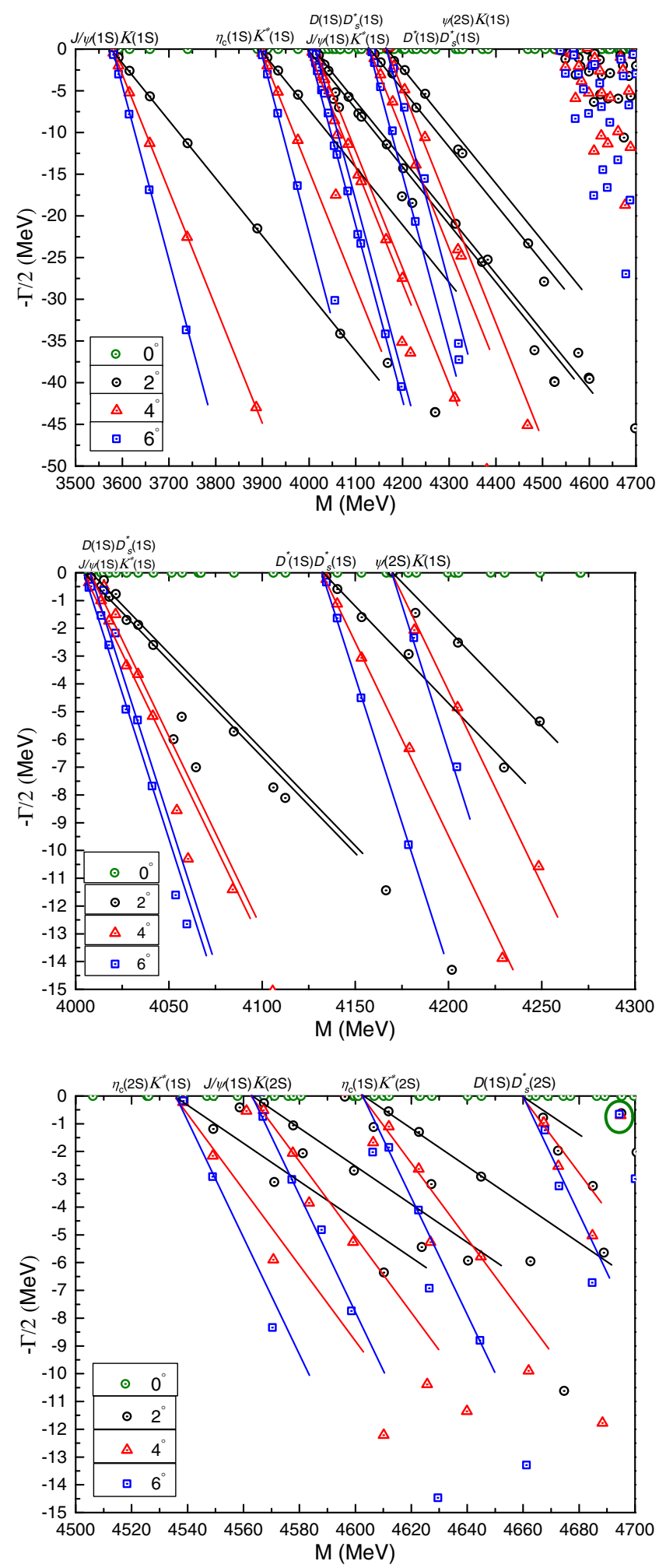

FIG. 7. Top panel: the complete coupled-channel calculation of the $c \bar{c} s \bar{q}$ tetraquark system with $J^{P}=1^{+}$quantum numbers. We use the complex-scaling method of the chiral quark model varying $\theta$ from $0^{\circ}$ to $6^{\circ}$. Middle panel: enlarged top panel, with real values of energy ranging from 4.00 to $4.30 \mathrm{GeV}$. Bottom panel: enlarged top panel, with real values of energy ranging from 4.50 to $4.70 \mathrm{GeV}$. 
TABLE IX. Lowest-lying $c \bar{c} s \bar{q}$ tetraquark states with $J^{P}=1^{+}$calculated within the real range formulation of the chiral quark model. The allowed meson-meson, diquark-antidiquark, and K-type configurations are listed in the first and fifth columns; when possible, the experimental value of the noninteracting meson-meson threshold is labeled in parentheses. Each channel is assigned an index in the second and sixth columns. The theoretical mass obtained in each channel is shown in the third and seventh columns, and the coupled result for each kind of configuration is presented in the fourth and last columns. When a complete coupled-channel calculation is performed, the last row of the table indicates the lowest-lying mass (unit, MeV).

\begin{tabular}{|c|c|c|c|c|c|c|c|}
\hline Channel & Index & $M$ & Mixed & Channel & Index & $M$ & Mixed \\
\hline$\left(\eta_{c} K^{*}\right)^{1}(3873)$ & 1 & 3896 & & $K_{1}$ & 19 & 4426 & \\
\hline$(J / \psi K)^{1}(3591)$ & 2 & 3578 & & $K_{1}$ & 20 & 4422 & \\
\hline$\left(J / \psi K^{*}\right)^{1}(3989)$ & 3 & 4004 & & $K_{1}$ & 21 & 4443 & \\
\hline$\left(D D_{s}^{*}\right)^{1}(3982)$ & 4 & 4012 & & $K_{1}$ & 22 & 4231 & \\
\hline$\left(D^{*} D_{s}\right)^{1}(3975)$ & 5 & 4006 & & $K_{1}$ & 23 & 4266 & \\
\hline$\left(D^{*} D_{s}^{*}\right)^{1}(4119)$ & 6 & 4132 & 3578 & $K_{1}$ & 24 & 4188 & 4186 \\
\hline$\left(\eta_{c} K^{*}\right)^{8}$ & 7 & 4448 & & $K_{2}$ & 25 & 4215 & \\
\hline$(J / \psi K)^{8}$ & 8 & 4424 & & $K_{2}$ & 26 & 4252 & \\
\hline$\left(J / \psi K^{*}\right)^{8}$ & 9 & 4434 & & $K_{2}$ & 27 & 4175 & \\
\hline$\left(D D_{s}^{*}\right)^{8}$ & 10 & 4403 & & $K_{2}$ & 28 & 4420 & \\
\hline$\left(D^{*} D_{s}\right)^{8}$ & 11 & 4401 & & $K_{2}$ & 29 & 4427 & \\
\hline$\left(D^{*} D_{s}^{*}\right)^{8}$ & 12 & 4361 & 4224 & $K_{2}$ & 30 & 4442 & 4168 \\
\hline$(c s)_{3}(\bar{c} \bar{q})_{3}^{*}$ & 13 & 4352 & & $K_{3}$ & 31 & 4283 & \\
\hline$(c s)_{3}^{*}(\bar{c} \bar{q})_{\overline{3}}$ & 14 & 4357 & & $K_{3}$ & 32 & 4306 & \\
\hline$(c s)_{3}^{*}(\bar{c} \bar{q})_{\overline{3}}^{*}$ & 15 & 4350 & & $K_{3}$ & 33 & 4340 & \\
\hline$(c s)_{6}(\bar{c} \bar{q})_{6}^{*}$ & 16 & 4342 & & $K_{3}$ & 34 & 4314 & \\
\hline$(c s)_{6}^{*}(\bar{c} \bar{q})_{\overline{6}}$ & 17 & 4339 & & $K_{3}$ & 35 & 4348 & \\
\hline \multirow[t]{7}{*}{$(c s)_{6}^{*}(\bar{c} \bar{q})_{6}^{*}$} & 18 & 4270 & 4184 & $K_{3}$ & 36 & 4349 & 4178 \\
\hline & & & & $K_{4}$ & 37 & 4309 & \\
\hline & & & & $K_{4}$ & 38 & 4319 & \\
\hline & & & & $K_{4}$ & 39 & 4362 & \\
\hline & & & & $K_{4}$ & 40 & 4327 & \\
\hline & & & & $K_{4}$ & 41 & 4353 & \\
\hline & & & & $K_{4}$ & 42 & 4349 & 4191 \\
\hline \multicolumn{4}{|c|}{ Complete coupled channels: } & & & & 3578 \\
\hline
\end{tabular}

TABLE X. The distance, in femtometers, between any two quarks of the $J^{P}=1^{+} c \bar{c} s \bar{q}$ tetraquark resonance state obtained in all exotic configurations' coupled-channel calculation. These resonances, which masses are around 3.9$4.2 \mathrm{GeV}$, are labeled in the first column.

\begin{tabular}{lcccccc}
\hline \hline State & $r_{\bar{c} c}$ & $r_{\bar{c} \bar{q}}$ & $r_{\bar{c} s}$ & $r_{c \bar{q}}$ & $r_{c s}$ & $r_{s \bar{q}}$ \\
\hline$Z_{c s}(3947)$ & 0.41 & 0.78 & 0.71 & 0.77 & 0.71 & 0.70 \\
$Z_{c s}(4038)$ & 0.35 & 0.95 & 0.87 & 0.95 & 0.87 & 0.87 \\
$Z_{c s}(4137)$ & 0.44 & 0.94 & 0.83 & 0.92 & 0.86 & 0.88 \\
$Z_{c s}(4199)$ & 0.58 & 0.80 & 0.67 & 0.74 & 0.73 & 0.87 \\
$Z_{c s}(4211)$ & 0.60 & 0.84 & 0.65 & 0.76 & 0.75 & 0.90 \\
\hline \hline
\end{tabular}

within a mass region $3.5-4.7 \mathrm{GeV}$, the scattering states of $J / \psi K^{(*)}, \eta_{c} K^{*}$, and $D^{(*)} D_{s}^{*}$ are clearly shown in the top panel in Fig. 7. One could see that, with the rotated angle $\theta$ varied from $0^{\circ}$ to $6^{\circ}$, the calculated complex energy dots always descend and basically align along the corresponding threshold lines. However, since there are dense distributions at around 4.1 and $4.6 \mathrm{GeV}$, enlarged parts on these two energy regions are presented in, respectively, the middle and bottom panels in Fig. 7. No resonance pole is obtained in the energy range $4.0-4.3 \mathrm{GeV}$ in the middle panel; however, one narrow resonance is found at higher energy, shown in the bottom panel. With a mass range from 4.5 to $4.7 \mathrm{GeV}$, four continuum (scattering) states of $\eta_{c} K^{*}, J / \psi K$, and $D D_{s}^{*}$ in radial excitations are well shown; meanwhile, a fixed pole is circled at $(4695,1.3) \mathrm{MeV}$. Accordingly, the $X(4685)$ state with $1^{+}$quantum numbers reported by the 
LHCb Collaboration [2] could be explained as a $D(1 S) D_{s}^{*}(2 S)(4695)$ resonance.

It is worth emphasizing herein that the resonances obtained in the different kinds of coupled-channel investigations are quite unstable and they easily decay to a meson-meson scattering state; e.g., the $Z_{c s}(3947)$, $Z_{c s}(4038)$, and $Z_{c s}(4137)$, which are obtained in a coupled-channel calculation with only exotic color structures, are unavailable in the complete coupled-channel case in Fig. 7.

\section{The $J^{P}=2^{+} c \bar{c} s \bar{q}$ tetraquark system}

Table VI lists the allowed 14 channels in the highest spin state of the $c \bar{c} s \bar{q}$ tetraquark system considered herein. Particularly, there are four meson-meson structures, two diquark-antidiquark structures, and eight K-type ones. In a real-range investigation, Table XI summarizes the calculated results of these channels. Therein, the physical channels are listed in the first column, and they are indexed in the following one. The theoretical mass of each channel and coupled result in each kind of configuration is listed in the third and fourth column, respectively. Meanwhile, the lowestlying mass of system in a complete coupled channel is listed in the last row in Table XI. Additionally, Figs. 8-10 show the coupled-channel results in a complex-range study, and the resonance states are indicated inside circles. When coupling is considered only for the exotic color configurations - the hidden-color channels of dimeson structures, diquark-antidiquark structures, and K-type ones-Table XII presents the exotic resonances whose masses are less than $4.3 \mathrm{GeV}$, and their inner structures are also analyzed. Further details are discussed in the following.

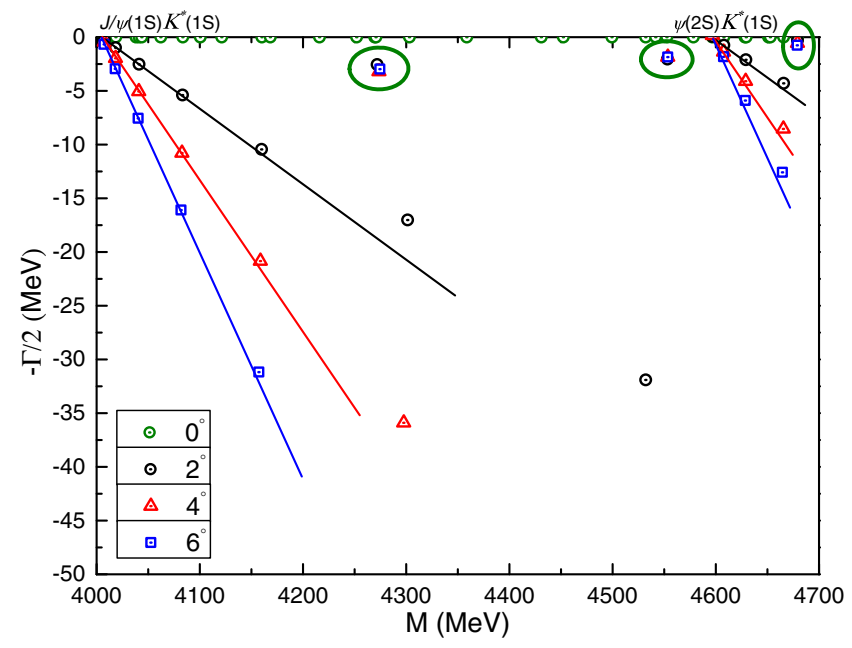

FIG. 8. The coupled-channel calculation of the $c \bar{c} s \bar{q}$ tetraquark system with $J^{P}=2^{+}$quantum numbers. Particularly, the $(c \bar{q})(s \bar{c})$ dimeson channels are excluded. We use the complexscaling method of the chiral quark model varying $\theta$ from $0^{\circ}$ to $6^{\circ}$.

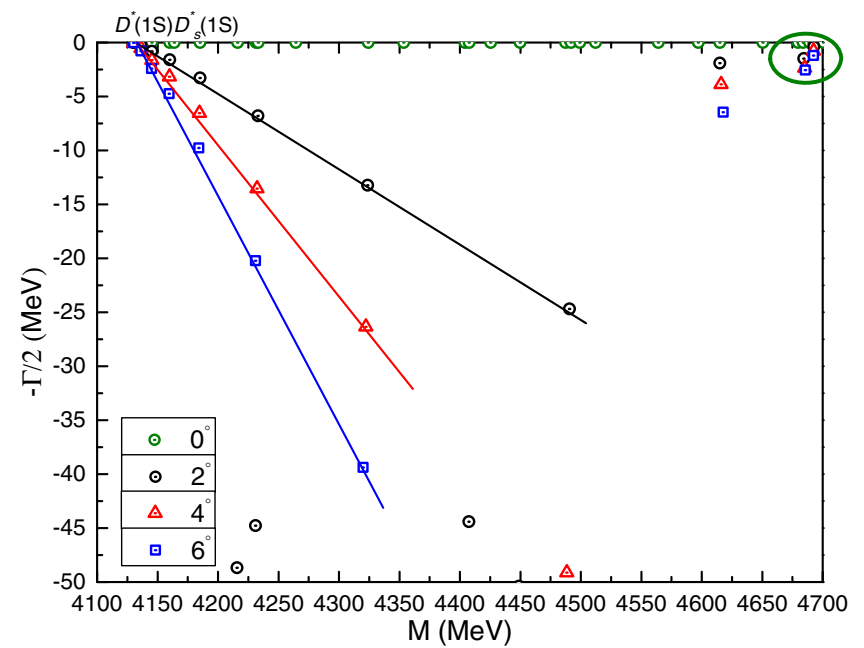

FIG. 9. The coupled-channel calculation of the $c \bar{c} s \bar{q}$ tetraquark system with $J^{P}=2^{+}$quantum numbers. Particularly, the $(c \bar{c})(s \bar{q})$ dimeson channels are excluded. We use the complexscaling method of the chiral quark model varying $\theta$ from $0^{\circ}$ to $6^{\circ}$.

Exotic states in $(c \bar{c})(s \bar{q})$ dimeson channels.-The calculated theoretical masses for the $J / \psi K^{*}$ in both color-singlet and -octet channels are 4004 and $4466 \mathrm{MeV}$, respectively (see Table XI). Therefore, the $\left(J / \psi K^{*}\right)^{1}$ state is of scattering nature and cannot be identified as the $Z_{c s}(4000)$ reported by the LHCb Collaboration.

Figure 8 illustrates the distribution of complex energies when a coupled-channel investigation that includes the $J \psi K^{*}$ dimeson, diquark-antidiquark, and $\mathrm{K}$-type structures is performed in the CSM. Within a mass region $4.0-4.7 \mathrm{GeV}$, the scattering states of $J / \psi(1 S) K^{*}(1 S)$ and $\psi(2 S) K^{*}(1 S)$ are well obtained. However, one could also realize that three narrow resonances, circled with green lines, are not sensitive

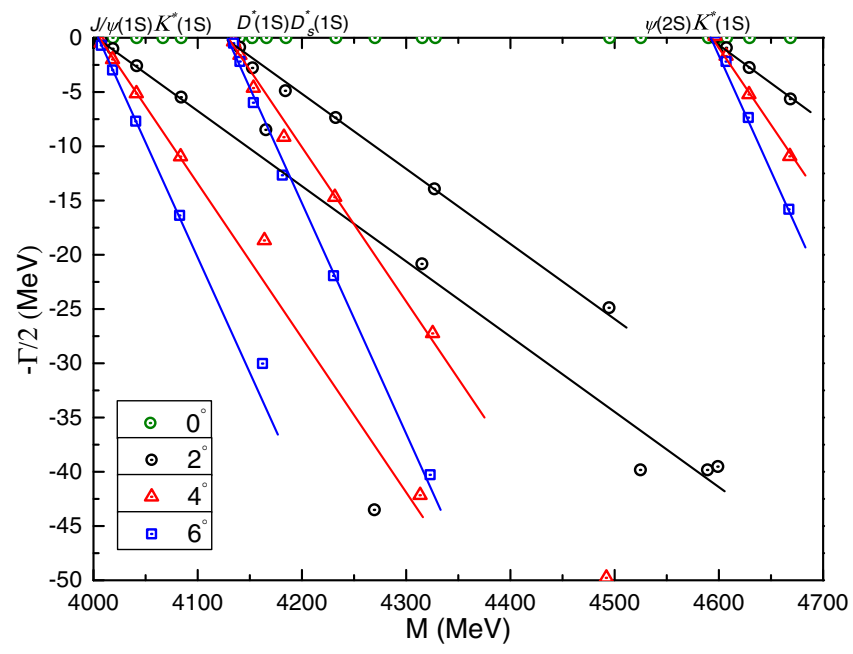

FIG. 10. The complete coupled-channel calculation of the $c \bar{c} s \bar{q}$ tetraquark system with $J^{P}=2^{+}$quantum numbers. We use the complex-scaling method of the chiral quark model varying $\theta$ from $0^{\circ}$ to $6^{\circ}$. 
TABLE XI. Lowest-lying $c \bar{c} s \bar{q}$ tetraquark states with $J^{P}=2^{+}$ calculated within the real range formulation of the chiral quark model. The allowed meson-meson, diquark-antidiquark, and K-type configurations are listed in the first column; when possible, the experimental value of the noninteracting meson-meson threshold is labeled in parentheses. Each channel is assigned an index in the second column. The theoretical mass obtained in each channel is shown in the third column, and the coupled result for each kind of configuration is presented in the last column. When a complete coupled-channel calculation is performed, the last row of the table indicates the lowest-lying mass (unit, MeV).

\begin{tabular}{lrrr}
\hline \hline Channel & Index & $M$ & Mixed \\
\hline$\left(J / \psi K^{*}\right)^{1}(3989)$ & 1 & 4004 & \\
$\left(D^{*} D_{s}^{*}\right)^{1}(4119)$ & 2 & 4132 & 4004 \\
$\left(J / \psi K^{*}\right)^{8}$ & 3 & 4466 & \\
$\left(D^{*} D_{s}^{*}\right)^{8}$ & 4 & 4411 & 4317 \\
$(c s)_{3}^{*}(\bar{c} \bar{q})_{3}^{*}$ & 5 & 4381 & \\
$(c s)_{6}^{*}(\bar{c} \bar{q})_{\frac{*}{6}}^{*}$ & 6 & 4346 & 4296 \\
$K_{1}$ & 7 & 4462 & \\
& 8 & 4296 & 4296 \\
$K_{2}$ & 9 & 4283 & \\
& 10 & 4461 & 4283 \\
$K_{3}$ & 11 & 4348 & \\
& 12 & 4373 & 4302 \\
$K_{4}$ & 13 & 4367 & \\
& 14 & 4378 & 4302 \\
Complete coupled channels: & & 4004 \\
\hline \hline
\end{tabular}

with respect to the variation of the complex angle $\theta$. The calculated masses and widths for these resonances are $(4271,5.2),(4553,4.0)$, and $(4678,1.6) \mathrm{MeV}$. In analogy with the $\psi(2 S) K^{*}(1 S)(4675)$ resonance obtained in the $0^{+}$ channel, the $\psi(2 S) K^{*}(1 S)(4678)$ resonance obtained here could also be related to the announced $X(4630)$, since the spin-parity of the $X(4685)$ state has been experimentally assigned to be $1^{+}$.

Exotic states in $(c \bar{q})(s \bar{c})$ dimeson channels.-Considering the $D^{*} D_{s}^{*}$ dimeson channel with quantum numbers $2^{+}$shown in Table XI, the lowest masses of color-singlet and hiddencolor structures are 4132 and $4411 \mathrm{MeV}$, respectively, and thus our conclusion on having scattering states in colorsinglet channel remains. Figure 9 shows the distribution of the complex energy dots obtained in a coupled-channel calculation with $D^{*} D_{s}^{*}$ dimeson configurations, diquarkantidiquark structures, and K-type arrangements. Parti cularly, the scattering nature of $D^{*}(1 S) D_{s}^{*}(1 S)$ is clearly shown in the 4.1-4.7 energy region. However, two quite close resonance poles are circled in the complex plane in Fig. 9. Therein, the two resonance states can be identified as $D^{*}(1 S) D_{s}^{*}(1 S)(4685)$ and $D^{*}(1 S) D_{s}^{*}(1 S)(4692)$, and their widths are 4.8 and $1.6 \mathrm{MeV}$, respectively. Although the mass of the $D^{*}(1 S) D_{S}^{*}(1 S)(4685)$ structure extremely coincides with that of the $X(4685)$ state, the spin-parity $2^{+}$is different from its experimental assignment.
TABLE XII. The distance, in femtometers, between any two quarks of the $J^{P}=2^{+} c \bar{c} s \bar{q}$ tetraquark resonance state obtained in all exotic configurations' coupled-channel calculation. These resonances, which masses are below $4.3 \mathrm{GeV}$, are labeled in the first column.

\begin{tabular}{lcccccc}
\hline \hline State & $r_{\bar{c} c}$ & $r_{\bar{c} \bar{q}}$ & $r_{\bar{c} s}$ & $r_{c \bar{q}}$ & $r_{c s}$ & $r_{s \bar{q}}$ \\
\hline$Z_{c s}(4145)$ & 0.41 & 0.96 & 0.88 & 0.95 & 0.88 & 0.88 \\
$Z_{c s}(4276)$ & 0.65 & 0.83 & 0.63 & 0.74 & 0.74 & 0.92 \\
\hline \hline
\end{tabular}

The fully coupled-channel case.-As shown in Table XI, the exotic configurations, which are diquark-antidiquark and K-type arrangements, lie generally in the mass range 4.3-4.4 GeV. In coupled-channel computations for each kind of configuration, the lowest-lying mass located at $4004 \mathrm{MeV}$ is still the theoretical value of the $\left(\mathrm{J} / \psi \mathrm{K}^{*}\right)^{1}$ threshold channel, and this fact is not changed in a fully coupled-channel calculation. Meanwhile, the other coupled-channel structures have higher masses at around $4.3 \mathrm{GeV}$.

When all of the exotic color structures are considered in a coupled-channel study, Table XII lists two resonance states whose mass is below $4.3 \mathrm{GeV}$. The distances between any two quarks are less than $1 \mathrm{fm}$, and, hence, they are good candidates of compact tetraquarks with color resonance structures.

In a further step, when the CSM is employed in a fully coupled-channel calculation, Fig. 10 shows the distribution of the scattering states for $J / \psi(1 S) K^{*}(1 S), D^{*}(1 S) D_{S}^{*}(1 S)$, and $\psi(2 S) K^{*}(1 S)$ in the mass region $4.0-4.7 \mathrm{GeV}$. As in the former cases, no bound state is found, and the calculated resonance states in different kinds of coupled-channel studies, e.g., $\psi(2 S) K^{*}(1 S)(4678), D^{*}(1 S) D_{s}^{*}(1 S)(4685)$, and $D^{*}$ $(1 S) D_{s}^{*}(1 S)(4692)$, etc., are quite unstable decaying easily to the $J / \psi K^{*}$ and $D^{*} D_{s}^{*}$ meson-meson scattering states.

\section{SUMMARY}

A systematical investigation of hidden-charm tetraquarks with strange content $c \bar{c} s \bar{q}(q=u, d)$ has been performed within a chiral quark model formalism. The model, which includes the one-gluon exchange, a linear-screened confining, and Goldstone-boson exchange interactions between quarks, has been successfully applied to the description of hadron, hadron-hadron, and multiquark phenomenology. In particular, the hidden-charm pentaquarks and doubly charmed tetraquark are well predicted in our previous theoretical investigations. Our formulation in real- and complex-scaling method of the theoretical formalism allows us to distinguish three kinds of scattering singularities: bound, resonance, and continuum. Furthermore, the mesonmeson, diquark-antidiquark, and K-type configurations, plus their couplings, are considered for the tetraquark system. Finally, the Rayleigh-Ritz variational method is employed in dealing with the spatial wave functions of the $c \bar{c} s \bar{q}$ tetraquark 
states, which are expanded by means of the well-known GEM in Ref. [59].

Generally, three kinds of computations are performed on the hidden-charm tetraquarks with strangeness whose quantum numbers are $J^{P}=0^{+}, 1^{+}$, and $2^{+}$states, namely, taking into account only $(c \bar{c})(s \bar{q})$ channels, just $(c \bar{q})(s \bar{c})$ ones, and a fully coupled-channel case. In order to present reasonable investigations on these three cases, complete color configurations of tetraquark systems are considered. Several resonances, either compact or loosely bound, are obtained in $(c \bar{c})(s \bar{q})$ and $(c \bar{q})(s \bar{c})$ channels; however, the same ones are quite unstable in a fully coupled-channel case. Each computation could separately contribute to disentangle the nature of the $Z_{c s}$ states because, from a theoretical point of view, they provide insights about making initial assumptions related with quark rearrangement and, from an experimental point of view, they warn the ease with which the $Z_{c s}$ states tend to disappear.

Our theoretical findings, compared with experimental observations, can be summarized as follows:

(i) In a coupled-channel computation, in which all exotic configurations are considered, the $Z_{c s}(3985)$ and $Z_{c s}(4000)$ could both be identified as compact $c \bar{c} s \bar{q}$ tetraquark states with $J^{P}=1^{+}$, and their sizes are less than $1 \mathrm{fm}$. (ii) In $(c \bar{c})(s \bar{q})$ or $(c \bar{q})(s \bar{c})$ dimeson channels, the $Z_{c s}(4220)$ could be compatible with a hadronic molecular resonance of either $\eta_{c}(2 S) K(1 S)(4255)$ with spin-parity $0^{+}$or $\psi(2 S) K(1 S)(4254)$ and $D^{*}(1 S) D_{s}^{*}(1 S)(4254)$ with $1^{+}$quantum numbers.

(iii) In a fully coupled-channel case, the $X(4685)$ could be associated with a hadronic molecular resonance whose structure resembles the $D(1 S) D_{S}^{*}(2 S)(4695)$ arrangement with quantum numbers $J^{P}=1^{+}$.

Finally, similar types of $c \bar{c} s \bar{q}$ tetraquark resonances are obtained in $0^{+}, 1^{+}$, and $2^{+}$channels for the energy ranges $[3.8,4.2],[4.1,4.3]$, and $[4.5,4.6] \mathrm{GeV}$, respectively. Their structure resembles either compact or molecular tetraquarks; however, most of them do not prevail when a complete coupled-channel calculation is performed.

\section{ACKNOWLEDGMENTS}

Work was partially financed by National Natural Science Foundation of China under Grants No. 11535005 and No. 11775118; the Zhejiang Provincial Natural Science Foundation under Grant No. Q22A053894; the Ministerio Español de Ciencia e Innovación, Grant No. PID2019107844 GB-C22; and Junta de Andalucía under Contract No. Operativo FEDER Andalucía 2014-2020 UHU1264517, P18-FR-5057, and PAIDI FQM-370.
[1] M. Ablikim et al. (BESIII Collaboration), Phys. Rev. Lett. 126, 102001 (2021).

[2] R. Aaij et al. (LHCb Collaboration), Phys. Rev. Lett. 127, 082001 (2021).

[3] Z. Yang, X. Cao, F.-K. Guo, J. Nieves, and M. P. Valderrama, Phys. Rev. D 103, 074029 (2021).

[4] L. Meng, B. Wang, and S.-L. Zhu, Phys. Rev. D 102, 111502 (2020).

[5] Z.-F. Sun and C.-W. Xiao, arXiv:2011.09404.

[6] B. Wang, L. Meng, and S.-L. Zhu, Phys. Rev. D 103, L021501 (2021).

[7] N. Ikeno, R. Molina, and E. Oset, Phys. Lett. B 814, 136120 (2021).

[8] Z.-M. Ding, H.-Y. Jiang, D. Song, and J. He, Eur. Phys. J. C 81, 732 (2021).

[9] Q.-N. Wang, W. Chen, and H.-X. Chen, Chin. Phys. C 45, 093102 (2021).

[10] Y.-J. Xu, Y.-L. Liu, C.-Y. Cui, and M.-Q. Huang, Phys. Rev. D 104, 094028 (2021).

[11] M.-J. Yan, F.-Z. Peng, M. S. Sánchez, and M. P. Valderrama, arXiv:2102.13058 [Phys. Rev. D (to be published)].

[12] Z.-H. Guo and J. A. Oller, Phys. Rev. D 103, 054021 (2021).

[13] R. Chen and Q. Huang, Phys. Rev. D 103, 034008 (2021).

[14] M.-Z. Liu, J.-X. Lu, T.-W. Wu, J.-J. Xie, and L.-S. Geng, arXiv:2011.08720.
[15] X. Jin, X. Liu, Y. Xue, H. Huang, and J. Ping, arXiv:2011.12230.

[16] M. Karliner and J. L. Rosner, Phys. Rev. D 104, 034033 (2021).

[17] B.-D. Wan and C.-F. Qiao, Nucl. Phys. B968, 115450 (2021).

[18] D. Ebert, R. N. Faustov, and V. O. Galkin, Eur. Phys. J. C 58, 399 (2008).

[19] R. N. Faustov, V. O. Galkin, and E. M. Savchenko, Universe 7, 94 (2021).

[20] Z.-G. Wang, Chin. Phys. C 45, 073107 (2021).

[21] J.-Z. Wang, Q.-S. Zhou, X. Liu, and T. Matsuki, Eur. Phys. J. C 81, 51 (2021).

[22] M.-C. Du, Q. Wang, and Q. Zhao, arXiv:2011.09225.

[23] X. Cao, J.-P. Dai, and Z. Yang, Eur. Phys. J. C 81, 184 (2021).

[24] U. Özdem and K. Azizi, Eur. Phys. J. Plus 136, 968 (2021).

[25] K. Azizi and N. Er, Eur. Phys. J. C 81, 61 (2021).

[26] J. Y. Süngü, A. Türkan, H. Sundu, and E. V. Veliev, arXiv: 2011.13013.

[27] H.-X. Chen, arXiv:2103.08586.

[28] L. Meng, B. Wang, G.-J. Wang, and S.-L. Zhu, Sci. Bull. 66, 2065 (2021).

[29] X.-D. Yang, F.-L. Wang, Z.-W. Liu, and X. Liu, Eur. Phys. J. C 81, 807 (2021).

[30] Z.-G. Wang, arXiv:2103.04236. 
[31] X. Chen, Y. Tan, and Y. Chen, Phys. Rev. D 104, 014017 (2021).

[32] J. F. Giron, R. F. Lebed, and S. R. Martinez, Phys. Rev. D 104, 054001 (2021).

[33] A. Türkan, J. Y. Süngü, and E. V. Veliev, arXiv:2103.05515.

[34] Y.-H. Ge, X.-H. Liu, and H.-W. Ke, Eur. Phys. J. C81, 854 (2021).

[35] U. Ozdem and A. K. Yildirim, Phys. Rev. D 104, 054017 (2021).

[36] G. Yang, J. Ping, and F. Wang, Phys. Rev. D 95, 014010 (2017).

[37] G. Yang, J. Ping, and J. Segovia, Phys. Rev. D 99, 014035 (2019).

[38] G. Yang, J. L. Ping, and J. Segovia, Phys. Rev. D 101, 074030 (2020).

[39] G. Yang, J. L. Ping, and J. Segovia, Phys. Rev. D 101, 014001 (2020).

[40] G. Yang, J. Ping, and J. Segovia, Phys. Rev. D 102, 054023 (2020).

[41] G. Yang, J. Ping, and J. Segovia, Phys. Rev. D 103, 074011 (2021).

[42] R. Aaij et al. (LHCb Collaboration), Phys. Rev. Lett. 115, 072001 (2015).

[43] R. Aaij et al. (LHCb Collaboration), Phys. Rev. Lett. 122, 222001 (2019).

[44] R. Aaij et al. (LHCb Collaboration), arXiv:2109.01038.

[45] R. Aaij et al. (LHCb Collaboration), arXiv:2109.01056.

[46] J. Segovia, A. M. Yasser, D. R. Entem, and F. Fernandez, Phys. Rev. D 78, 114033 (2008).

[47] J. Segovia, D. R. Entem, F. Fernandez, and E. Hernandez, Int. J. Mod. Phys. E 22, 1330026 (2013).

[48] J. Segovia, P. G. Ortega, D. R. Entem, and F. Fernández, Phys. Rev. D 93, 074027 (2016).

[49] G. Yang, J. Ping, P. G. Ortega, and J. Segovia, Chin. Phys. C 44, 023102 (2020).

[50] J. Segovia, A. M. Yasser, D. R. Entem, and F. Fernandez, Phys. Rev. D 80, 054017 (2009).
[51] J. Segovia, D. Entem, and F. Fernandez, Phys. Rev. D 83, 114018 (2011).

[52] J. Segovia, D. R. Entem, and F. Fernández, Phys. Lett. B 715, 322 (2012).

[53] J. Segovia, D. R. Entem, and F. Fernandez, Phys. Rev. D 91, 094020 (2015).

[54] P. G. Ortega, J. Segovia, D. R. Entem, and F. Fernandez, Phys. Rev. D 81, 054023 (2010).

[55] P. G. Ortega, J. Segovia, D. R. Entem, and F. Fernandez, Phys. Rev. D 94, 074037 (2016).

[56] P. G. Ortega, J. Segovia, D. R. Entem, and F. Fernández, Phys. Rev. D 95, 034010 (2017).

[57] P. G. Ortega, J. Segovia, D. R. Entem, and F. Fernandez, Eur. Phys. J. C 80, 223 (2020).

[58] G. Yang, J. Ping, and J. Segovia, Symmetry 12, 1869 (2020).

[59] E. Hiyama, Y. Kino, and M. Kamimura, Prog. Part. Nucl. Phys. 51, 223 (2003).

[60] J. Aguilar and J. M. Combes, Commun. Math. Phys. 22, 269 (1971).

[61] E. Balslev and J. M. Combes, Commun. Math. Phys. 22, 280 (1971).

[62] J. Vijande, F. Fernandez, and A. Valcarce, J. Phys. G 31, 481 (2005).

[63] M. D. Scadron, Phys. Rev. D 26, 239 (1982).

[64] R. Garcia-Martin, R. Kaminski, J. R. Pelaez, and J. Ruiz de Elvira, Phys. Rev. Lett. 107, 072001 (2011).

[65] M. Albaladejo and J. A. Oller, Phys. Rev. D 86, 034003 (2012).

[66] J. R. Pelaez, Phys. Rep. 658, 1 (2016).

[67] G. S. Bali, H. Neff, T. Duessel, T. Lippert, and K. Schilling (SESAM Collaboration), Phys. Rev. D 71, 114513 (2005).

[68] J. Segovia, D. R. Entem, and F. Fernandez, Phys. Lett. B 662, 33 (2008).

[69] J. D. Weinstein and N. Isgur, Phys. Rev. Lett. 48, 659 (1982). 